\title{
A wave-based numerical scheme for damage detection and identification in two-dimensional composite structures
}

\author{
R.K. Apalowo ${ }^{\mathrm{a}}$, D. Chronopoulos ${ }^{\mathrm{a}}$ \\ ${ }^{a}$ Institute for Aerospace Technology \& The Composites Research Group, The University of Nottingham, NG7 2RD, \\ $U K$
}

\begin{abstract}
Previous studies on wave inspection in different propagation directions have focussed on the analysis of wave propagation and wave scattering from various types of joints in two-dimensional monolayered structures. In this work, a Finite Element (FE) based numerical scheme is presented for quantifying wave interaction with localised structural damage within two-dimensional layered composite structures having arbitrary layering, complexities and material characteristics. The scheme discretise a damaged structural medium into a system of $N$ healthy substructures (waveguides) connected through a joint which bears the localised structural damage/discontinuity. Wave propagation constants along different propagation directions of the substructures are sought by combining Periodic Structure Theory (PST) and the FE method. The damaged joint is modelled using standard FE approach, ensuring joint-substructures mesh conformity. This is coupled to the obtained wave propagation constants in order to determine scattering coefficients for the wave interaction with damage in different propagation directions within the structure. Wave interaction coefficients for different damage types and structural parameters are analysed in order to establish an optimum basis for detecting and identifying damage, as well as assessing the orientation and extent of the detected damage. The main advantage of this scheme is precise predictions at a very low computational cost.
\end{abstract}

Keywords: Periodic structure theory, Wave finite element method, Wave scattering from damage, Two-dimensional structures, Composite structures

Email address: Rilwan. Apalowo@nottingham.ac.uk (R.K. Apalowo) 


\section{Nomenclature}

a Wave amplitude

c Wave scattering coefficient

D, $\mathbb{D} \quad$ Dynamic stiffness matrices of the waveguide and the coupling joint

$\mathbf{f}^{\mathrm{e}} \quad$ Vector of forces applied to the coupling joint at its interface with connected waveguides

k Wavenumber

$\mathbf{K}, \mathbf{M}, \mathbf{C}$ Stiffness, mass and damping matrices of a waveguide's modelled segment

q, $\mathbf{f} \quad$ Physical displacement and forcing vectors for a waveguide

R Rotation matrices of the system's waveguides

S Wave scattering matrix

$\mathbf{T} \quad$ Wave propagation transfer matrix

$\mathbf{z} \quad$ Physical displacement vector for the coupling joint

$\boldsymbol{\phi}, \boldsymbol{\Phi}$ Eigenvector and grouped eigenvector

$\lambda \quad$ Propagation constant and eigenvalue of the wave propagation eigenproblem

$\mathbb{K}, \mathbb{M}, \mathbb{C}$ Stiffness, mass and damping matrices of the coupling joint

$\mathbb{R}_{\mathrm{T}}, \mathbb{R}_{\mathrm{T}}^{*} \quad$ Transformation matrix for the coupling joint's displacement and force vectors

$\omega \quad$ Circular frequency 


\begin{tabular}{|c|c|}
\hline $\mathfrak{R}$ & Real operator \\
\hline$\varepsilon, \mu$ & Wave propagation constants for the waveguide and the coupling joint \\
\hline$b, h$ & width and depth of a cross-section \\
\hline$E, G, v$, & Elastic modulus, shear modulus, Poisson's ratio and density of an elastic waveguide \\
\hline$i$ & Imaginary unit \\
\hline$j$ & $\begin{array}{l}\text { Number of DoF on each periodic cross-section of the waveguide's segment, along } \\
\text { the axis in which wave properties are sought }\end{array}$ \\
\hline$L$ & Length \\
\hline$n$ & Number of periodic layers along the axis of wave imposition \\
\hline$R_{T}, R_{T}^{*}$ & Transformation matrix for the waveguide's displacement and force vectors \\
\hline \multicolumn{2}{|c|}{ Subscripts } \\
\hline ¿, n & $\begin{array}{l}\text { property of interface and non-interface nodes of the coupling joint with the waveg- } \\
\text { uides }\end{array}$ \\
\hline$J, \sqrt{ }$ & Property of a coupling joint \\
\hline$k, n$ & Waveguide indices \\
\hline$L, R, I$ & Left, right sides and interior indices \\
\hline \multicolumn{2}{|r|}{$\begin{array}{c}L T, I T, R T, L I, I, R I, L B, I B, R B \text { left top, internal top, right top, left internal, internal, right } \\
\text { internal, left base, internal base and right base of a modelled segment }\end{array}$} \\
\hline$N$ & Waveguide indices and total number of waveguides existing in the considered system \\
\hline$q, \mathrm{f}$ & Displacement and forcing indices \\
\hline
\end{tabular}




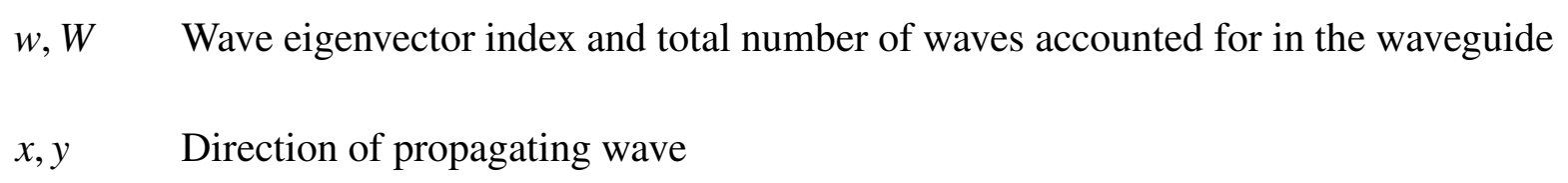

\section{Superscripts}

inc, ref Positive and negative going waves properties

$g \quad$ Global coordinate index

$s \quad$ Periodic segment positioning index

\section{Introduction}

Composite structures, in recent years, have seen growing applications in the automotive, construction and aerospace industries mainly due to their great mechanical characteristics over other material structures. As result of the increased applications, inspection of the structures to ensure continuous use has also received great interest. Most composite structures such as plates and shells can be regarded as two-dimensional structures due to the propagation behaviour of waves travelling through them. In these structures, waves travel in two directions, and when they interact with any form of discontinuity (such as damage or change in cross-section) within the structure, they transmit through or reflect from the discontinuity also in two directions. This therefore gives rise to the need for studying wave interaction with damage in every propagation direction of the structure.

Typically, cost of inspecting and repairing damage in aircraft structures amounts to about onethird of its lifecycle [1]. Offline inspection could lead to a significant financial loss due to massive unavailability of the structure during the inspection process. As a result of this, Non-Destructive Evaluation (NDE) techniques for monitoring and evaluation of damage have thus become important for low-cost and efficient inspection of in-service structures. Various techniques have been developed and implemented towards that goal. Of these techniques, the vibration-based methods are among the most widely employed due to their comparative advantages $[2,3]$. They are 
based on extraction of vibration response to determine damage parameters. For instance, In [4], vibration-based methodology is developed which calculates damage index based on the analysis of Poincare maps to determine presence and the location of a damage within plates. Theoretically, changes in structural properties, due to damage for example, will lead to changes in the modal properties such as natural frequencies [5, 6], mode shapes [7] and stiffness [8] of the structure. A major limitation of this approach is that it is mostly effective in detecting the presence of damage while determining the mode, location and extent of damage is a difficult (if not impossible) process at best. However, localising damage is prevalent and critical in structural integrity evaluation. Of all the NDE techniques, such as electromagnetic testing, laser testing, thermal testing, etc. [9], suitable for detecting localised damage, the ultrasonic testing is of great interest due to its ability to induce accurate localised damage detection at high frequencies. When a wave propagates through a medium encounters a damage, it is reflected, or transmitted. The reflection and transmission coefficients (known as the scattering coefficients) obtained from the wave-damage interaction is an underlying parameters for damage detection in ultrasonic testing [10]. As established in [1113], the scattering coefficients greatly depend on damage type. Hence, wave interaction scattering coefficients can be utilised for damage identification, as will be demonstrated in this study.

Advancements in computational tools development has resulted in significant increase in the application of model-based approaches to detect, analyse and classify damage in structures. Implementing a suitable modelling technique for this purpose has hence become an important aspect of Structural Health Monitoring (SHM). The Finite Element (FE) method is one of the most commonly employed modelling techniques. However, to detect small sized damage, especially at high frequency, a very fine mesh density is needed which results into a very large sized model. This makes FE method computationally expensive for this purpose. Wave-based modelling techniques are computationally affordable, especially for simple structures, because they require low computational resources. Thus, they are preferred for problems where FE method becomes computationally large. For arbitrarily complex structures, such as composite structures or structural networks, a Wave Finite Element (WFE) approach was introduced in $[14,15]$. The approach combines the periodic structure theory (PST) with FE to obtain wave propagation constants within a broad frequency range. WFE is extended to two-dimensional structures in [16]. It has been applied to a 
variety of structural situations, such as flat [17], curved [18] and stiffened [19] structures, under free [15, 20], forced [21-23] and simply supported [24] boundary conditions. It has also been applied to obtain wave scattering properties in cylindrical pipes with defects [25], structures with different types of joints [26-28] and damaged stiffened panels [29]. The approach has recently found applications in predicting the vibroacoustic and dynamic performance of composite panels $[30,31]$. The variability of vibroacoustic transmission through layered structures [32] as well as structural identification [33] have been investigated through the same methodology.

WFE method has shown great efficiency and versatility in its applications to various structural problems. However, very few of these applications considered two-dimensional structures. Wave propagation in two-dimensional structures using the WFE method was introduced in [16]. It was extended in [27], using the hybrid WFE-FE approach [26], to compute wave scattering from different joints in a coupled monolayered structures. In order to implement damage and compute wave scattering from the damage, the coupled structures have to be multilayered.

The principal contribution of this work is development of a WFE based numerical scheme for quantifying wave interaction with localised structural damage within two-dimensional multilayer composite structures having arbitrary layering, complexities and material characteristics. The scheme discretises a damaged structural medium into a system of $N$ healthy substructures (waveguides) connected through a joint which bears the localised structural damage/discontinuity. Wave propagation constants along different directions of the substructures are sought by combining periodic structure theory with the FE method. The damaged joint is modelled using a standard FE approach, ensuring joint-substructures mesh conformity. This is coupled to the obtained wave propagation constants in order to determine scattering coefficients of the wave interaction with damage in different propagation directions of the structure. Wave interaction coefficients for different damage types and parameters are analysed in order to establish an optimum basis for detecting and identifying damage, as well as assessing the orientation and extent of damage.

The remainder of this article is organized as follows: In Sec. 2, the computation of wave propagation constants in two-dimensional layered waveguide is presented. Sec. 3 presents numerical methodologies for damage implementation in structural segment. Wave interaction with structural damage and the computation of the interaction coefficients are presented in Sec. 4. Sec. 5 presents 
numerical case studies and concluding remarks on the presented work are given in Sec. 6 .

\section{Wave propagation in two-dimensional composite structure}

\subsection{Statement of the problem}

Elastic wave propagation is considered in the $x$ and $y$ directions of an arbitrarily layered composite waveguide (Fig. 1). A segment of the waveguide is modelled using a commercial FE software.

[Figure 1 about here.]

The stiffness, mass and damping matrices of the segment $\mathbf{K}, \mathbf{M}$ and $\mathbf{C}$ are extracted using classical FEM algorithm and the nodal displacements vector $\mathbf{q}$ is reordered according to a predefined sequence such as

$$
\mathbf{q}=\left\{\begin{array}{llllllllllll}
\mathbf{q}_{\mathrm{LT}} & \mathbf{q}_{\mathrm{IT}} & \mathbf{q}_{\mathrm{RT}} & \mathbf{q}_{\mathrm{L} I 1} & \mathbf{q}_{\mathrm{I} 1} & \mathbf{q}_{\mathrm{RI} 1} & \mathbf{q}_{\mathrm{L} 12} & \mathbf{q}_{\mathrm{I} 2} & \mathbf{q}_{\mathrm{R} I 2} & \mathbf{q}_{\mathrm{LB}} & \mathbf{q}_{\mathrm{IB}} & \mathbf{q}_{\mathrm{RB}}
\end{array}\right\}^{\top}
$$

where I corresponds to internal, LI,RI the interface edge and LT, RT, LB, RB the interface corner indices.

\subsection{PST formulation of the waveguide's equation of motion}

The time harmonic equation of motion of the modelled segment, assuming uniform and structural damping for all the DoFs, can be written as

$$
\left[\mathbf{K}+\mathrm{i} \omega \mathbf{C}-\omega^{2} \mathbf{M}\right] \mathbf{q}=\mathbf{f}
$$

where $\omega$ is the circular frequency and $\mathbf{f}$ is the nodal forces vector. The dynamic stiffness matrix can then be expressed as

$$
\mathbf{D}=\mathbf{K}+\mathrm{i} \omega \mathbf{C}-\omega^{2} \mathbf{M}
$$

The equation of motion (Eq. (2)) is therefore expressed as 


$$
\mathbf{D q}=\mathbf{f}
$$

A practical procedure for extracting the wave propagation characteristics of the segment is injecting a set of assumed propagation constants $\varepsilon_{x}$ or $\varepsilon_{y}$. The set of these constants can be chosen in relation to the direction of propagation towards which the wavenumbers are to be sought and according to the desired resolution of the wavenumber curves. In this study, propagation constants are injected along $y$ axis and propagation characteristics are sought along $x$ axis by transforming Eq. (4) into a standard eigenvalue problem with $x$ as the direction of wave propagation.

Applying the Bloch's theorem under the assumption of a time harmonic response, the displacements at each periodic edge along the axis of wave injection ( $y$ axis) can be expressed as a function of the displacements at one edge. Using the edge with indices LB, IB and RB as the edge of reference, then

$$
\begin{array}{ccc}
\mathbf{q}_{\mathrm{LT}}=\mathrm{e}^{-\mathrm{i} \varepsilon_{y}} \mathbf{q}_{\mathrm{LB}}, & \mathbf{q}_{\mathrm{IT}}=\mathrm{e}^{-\mathrm{i} \varepsilon_{y}} \mathbf{q}_{\mathrm{IB}}, & \mathbf{q}_{\mathrm{RT}}=\mathrm{e}^{-\mathrm{i} \varepsilon_{y}} \mathbf{q}_{\mathrm{RB}} \\
\mathbf{q}_{\mathrm{LI} 1}=\mathrm{e}^{-\mathrm{i} \frac{2}{3} \varepsilon_{y}} \mathbf{q}_{\mathrm{LB}}, & \mathbf{q}_{\mathrm{I} 1}=\mathrm{e}^{-\mathrm{i} \frac{2}{3} \varepsilon_{y}} \mathbf{q}_{\mathrm{IB}}, & \mathbf{q}_{\mathrm{RI} 1}=\mathrm{e}^{-\mathrm{i} \frac{2}{3} \varepsilon_{y}} \mathbf{q}_{\mathrm{RB}} \\
\mathbf{q}_{\mathrm{LI} 2}=\mathrm{e}^{-\mathrm{i} \frac{1}{3} \varepsilon_{y}} \mathbf{q}_{\mathrm{LB}}, & \mathbf{q}_{\mathrm{I} 2}=\mathrm{e}^{-\mathrm{i} \frac{1}{3} \varepsilon_{y}} \mathbf{q}_{\mathrm{IB}}, & \mathbf{q}_{\mathrm{RI} 2}=\mathrm{e}^{-\mathrm{i} \frac{1}{3} \varepsilon_{y}} \mathbf{q}_{\mathrm{RB}}
\end{array}
$$

Using same theorem, the corresponding force vectors can be expressed as

$$
\begin{aligned}
& \mathbf{f}_{\mathrm{LT}}=\mathrm{e}^{-\mathrm{i} \varepsilon_{y}} \mathbf{f}_{\mathrm{LB}}, \quad \mathbf{f}_{\mathrm{IT}}=\mathrm{e}^{-\mathrm{i} \varepsilon_{y}} \mathbf{f}_{\mathrm{IB}}, \quad \mathbf{f}_{\mathrm{RT}}=\mathrm{e}^{-\mathrm{i} \varepsilon_{y}} \mathbf{f}_{\mathrm{RB}} \\
& \mathbf{f}_{\mathrm{LI} 1}=\mathrm{e}^{-\mathrm{i} \frac{2}{3} \varepsilon_{y}} \mathbf{f}_{\mathrm{LB}}, \quad \mathbf{f}_{\mathrm{I} 1}=\mathrm{e}^{-\mathrm{i} \frac{2}{3} \varepsilon_{y}} \mathbf{f}_{\mathrm{IB}}, \quad \mathbf{f}_{\mathrm{RI} 1}=\mathrm{e}^{-\mathrm{i} \frac{2}{3} \varepsilon_{y}} \mathbf{f}_{\mathrm{RB}} \\
& \mathbf{f}_{\mathrm{LI} 2}=\mathrm{e}^{-\mathrm{i} \frac{1}{3} \varepsilon_{y}} \mathbf{f}_{\mathrm{LB}}, \quad \mathbf{f}_{\mathrm{I} 2}=\mathrm{e}^{-\mathrm{i} \frac{1}{3} \varepsilon_{y}} \mathbf{f}_{\mathrm{IB}}, \quad \mathbf{f}_{\mathrm{RI} 2}=\mathrm{e}^{-\mathrm{i} \frac{1}{3} \varepsilon_{y}} \mathbf{f}_{\mathrm{RB}}
\end{aligned}
$$

where $\varepsilon_{y}$, the propagation constant in the $y$ direction, is directly related to the wavenumber $k_{y}$ through the relation

$$
\varepsilon_{y}=k_{y} L_{y}
$$

Expressing Eq. (5) in tensorial form gives

$$
\mathbf{q}=\mathbf{R}_{\mathrm{T}}\left\{\begin{array}{lll}
\mathbf{q}_{\mathrm{LB}} & \mathbf{q}_{\mathrm{IB}} & \mathbf{q}_{\mathrm{RB}}
\end{array}\right\}^{\top}
$$


where

$$
\mathbf{R}_{\mathrm{T}}=\left[\begin{array}{ccc}
\lambda_{y} \mathbf{I} & \mathbf{0} & \mathbf{0} \\
\mathbf{0} & \lambda_{y} \mathbf{I} & \mathbf{0} \\
\mathbf{0} & \mathbf{0} & \lambda_{y} \mathbf{I} \\
\lambda_{y}^{\frac{2}{3}} \mathbf{I} & \mathbf{0} & \mathbf{0} \\
\mathbf{0} & \lambda_{y}^{\frac{2}{3}} \mathbf{I} & \mathbf{0} \\
\mathbf{0} & \mathbf{0} & \lambda_{y}^{\frac{2}{3}} \mathbf{I} \\
\lambda_{y}^{\frac{1}{3}} \mathbf{I} & \mathbf{0} & \mathbf{0} \\
\mathbf{0} & \lambda_{y}^{\frac{1}{3}} \mathbf{I} & \mathbf{0} \\
\mathbf{0} & \mathbf{0} & \lambda_{y}^{\frac{1}{3}} \mathbf{I} \\
\mathbf{I} & \mathbf{0} & \mathbf{0} \\
\mathbf{0} & \mathbf{I} & \mathbf{0} \\
\mathbf{0} & \mathbf{0} & \mathbf{I}
\end{array}\right]
$$

with

$$
\lambda_{y}=\mathrm{e}^{-\mathrm{i} \varepsilon_{y}}
$$

$\mathbf{R}_{\mathrm{T}}$ is the transformation matrix for expressing the displacements at each periodic edge as a function of the displacements at the reference edge.

Assuming equilibrium along the reference edge, Eq. (6) can also be expressed in tensorial form as

$$
\mathbf{R}_{\mathrm{T}}^{*} \mathbf{f}=\left\{\begin{array}{lll}
\mathbf{f}_{\mathrm{LB}} & \mathbf{f}_{\mathrm{IB}} & \mathbf{f}_{\mathrm{RB}}
\end{array}\right\}^{\top}
$$

where $\mathbf{R}_{\mathrm{T}}^{*}$, which is a Hermitian transpose of $\mathbf{R}_{\mathrm{T}}$, is the transformation matrix for the forces vector.

Substituting Eqs. (8) and (11) into Eq. (4) gives a reformulated standard equation of motion as

$$
\left[\mathbf{R}_{\mathrm{T}}^{*} \mathbf{K R}_{\mathrm{T}}+\mathrm{i} \omega \mathbf{R}_{\mathrm{T}}^{*} \mathbf{C R}_{\mathrm{T}}-\omega^{2} \mathbf{R}_{\mathrm{T}}^{*} \mathbf{M} \mathbf{R}_{\mathrm{T}}\right]\left\{\begin{array}{c}
\mathbf{q}_{\mathrm{LB}} \\
\mathbf{q}_{\mathrm{IB}} \\
\mathbf{q}_{\mathrm{RB}}
\end{array}\right\}=\left\{\begin{array}{c}
\mathbf{f}_{\mathrm{LB}} \\
\mathbf{f}_{\mathrm{IB}} \\
\mathbf{f}_{\mathrm{RB}}
\end{array}\right\}
$$


The modelled periodic segment, as shown in Fig. 1, has three periodic segments along the axis of wave injection (i.e. $y$ axis). Generally, for a periodic segment with $n$ periodic layers along the axis of wave injection, the transformation matrix is expressed as

$$
\mathbf{R}_{\mathrm{T}}=\left[\begin{array}{ccc}
\lambda_{y}^{\frac{n}{n}} \mathbf{I} & \mathbf{0} & \mathbf{0} \\
\mathbf{0} & \lambda_{y}^{\frac{n}{n}} \mathbf{I} & \mathbf{0} \\
\mathbf{0} & \mathbf{0} & \lambda_{y}^{\frac{n}{n}} \mathbf{I} \\
\lambda_{y}^{\frac{n-1}{n}} \mathbf{I} & \mathbf{0} & \mathbf{0} \\
\mathbf{0} & \lambda_{y}^{\frac{n-1}{n}} \mathbf{I} & \mathbf{0} \\
\mathbf{0} & \mathbf{0} & \lambda_{y}^{\frac{n-1}{n}} \mathbf{I} \\
\lambda_{y}^{\frac{n-2}{n}} \mathbf{I} & \mathbf{0} & \mathbf{0} \\
\mathbf{0} & \lambda_{y}^{\frac{n-2}{n}} \mathbf{I} & \mathbf{0} \\
\mathbf{0} & \mathbf{0} & \lambda_{y}^{\frac{n-2}{n}} \mathbf{I} \\
\vdots & \ddots & \vdots \\
\lambda_{y}^{\frac{0}{n}} \mathbf{I} & \mathbf{0} & \mathbf{0} \\
\mathbf{0} & \lambda_{y}^{\frac{0}{n}} \mathbf{I} & \mathbf{0} \\
\mathbf{0} & \mathbf{0} & \lambda_{y}^{\frac{0}{n}} \mathbf{I}
\end{array}\right]
$$

\subsection{Formulation of eigenvalue problem}

The standard eigenvalue relation for the equation of motion (Eq. 12) is hereby formulated using a WFE approach which couples the periodic structure theory with the FE method.

In Eq. (12), $x$ is the direction along which propagation characteristics are sought when wave properties along $y$ are known and imposed as earlier informed. Hence, the dynamic stiffness matrix of the segment can be partitioned with regard to its left $L B$ (denoted as $L$ ), right $R B$ (denoted as $R$ ) and internal $I B$ (denoted as $I)$ DoF as

$$
\left[\begin{array}{ccc}
\mathbf{D}_{\mathrm{LL}} & \mathbf{D}_{\mathrm{LI}} & \mathbf{D}_{\mathrm{LR}} \\
\mathbf{D}_{\mathrm{IL}} & \mathbf{D}_{\mathrm{II}} & \mathbf{D}_{\mathrm{IR}} \\
\mathbf{D}_{\mathrm{RL}} & \mathbf{D}_{\mathrm{RI}} & \mathbf{D}_{\mathrm{RR}}
\end{array}\right]\left\{\begin{array}{c}
\mathbf{q}_{\mathrm{L}} \\
\mathbf{q}_{\mathrm{I}} \\
\mathbf{q}_{\mathrm{R}}
\end{array}\right\}=\left\{\begin{array}{c}
\mathbf{f}_{\mathrm{L}} \\
\mathbf{f}_{\mathrm{I}} \\
\mathbf{f}_{\mathrm{R}}
\end{array}\right\}
$$


It is assumed that no external forces are applied on the internal nodes, $\mathbf{f}_{\mathrm{I}}=\mathbf{0}$. Applying a dynamic condensation technique on the internal nodes DoF, Eq. (14) can be expressed as

$$
\left[\begin{array}{ll}
\mathbf{D}_{11} & \mathbf{D}_{12} \\
\mathbf{D}_{21} & \mathbf{D}_{22}
\end{array}\right]\left\{\begin{array}{c}
\mathbf{q}_{\mathrm{L}} \\
\mathbf{q}_{\mathrm{R}}
\end{array}\right\}=\left\{\begin{array}{c}
\mathbf{f}_{\mathrm{L}} \\
\mathbf{f}_{\mathrm{R}}
\end{array}\right\}
$$

with

$$
\begin{array}{ll}
\mathbf{D}_{11}=\mathbf{D}_{\mathrm{LL}}-\mathbf{D}_{\mathrm{LI}} \mathbf{D}_{\mathrm{II}}^{-1} \mathbf{D}_{\mathrm{IL}}, & \mathbf{D}_{12}=\mathbf{D}_{\mathrm{LR}}-\mathbf{D}_{\mathrm{LI}} \mathbf{D}_{\mathrm{II}}^{-1} \mathbf{D}_{\mathrm{IR}} \\
\mathbf{D}_{21}=\mathbf{D}_{\mathrm{RL}}-\mathbf{D}_{\mathrm{RI}} \mathbf{D}_{\mathrm{II}}^{-1} \mathbf{D}_{\mathrm{IL}}, & \mathbf{D}_{22}=\mathbf{D}_{\mathrm{RR}}-\mathbf{D}_{\mathrm{RI}} \mathbf{D}_{\mathrm{II}}^{-1} \mathbf{D}_{\mathrm{IR}}
\end{array}
$$

As earlier stated, it is assumed that no external forces are applied on the segment. As a result of this, the displacement continuity and equilibrium of forces equations at the interface of two consecutive periodic segments $s$ and $s+1$ are given as

$$
\begin{gathered}
\mathbf{q}_{\mathrm{L}}^{s+1}=\mathbf{q}_{\mathrm{R}}^{s} \\
\mathbf{f}_{\mathrm{L}}^{s+1}=-\mathbf{f}_{\mathrm{R}}^{s}
\end{gathered}
$$

Combining Eqs. (15) and (17), the relation of the displacements and forces of the two consecutive periodic segments can be expressed in the form

$$
\left\{\begin{array}{c}
\mathbf{q}_{\mathrm{L}}^{s+1} \\
\mathbf{f}_{\mathrm{L}}^{s+1}
\end{array}\right\}=\mathbf{T}\left\{\begin{array}{c}
\mathbf{q}_{\mathrm{L}}^{s} \\
\mathbf{f}_{\mathrm{L}}^{s}
\end{array}\right\}
$$

and the expression of the symplectic transfer matrix $\mathbf{T}$ is defined as

$$
\mathbf{T}=\left[\begin{array}{cc}
-\mathbf{D}_{12}^{-1} \mathbf{D}_{11} & \mathbf{D}_{12}^{-1} \\
-\mathbf{D}_{21}+\mathbf{D}_{22} \mathbf{D}_{12}^{-1} \mathbf{D}_{11} & -\mathbf{D}_{22} \mathbf{D}_{12}^{-1}
\end{array}\right]_{[2 j \times 2 j]}
$$

where $2 j$ is the number of DoF on each periodic cross-section (along $y$-axis) of the periodic waveguide's segment (Fig. 1).

As previously stated, propagation characteristics along $y$ direction are assumed known. The unknown propagation constant along $x$ direction $\lambda_{x}=\mathrm{e}^{-\mathrm{i} k_{x} L_{x}}$ relates the right and left nodal displacements and forces according to Bloch's theorem as 


$$
\begin{gathered}
\mathbf{q}_{\mathrm{R}}^{s}=\lambda_{\mathrm{x}} \mathbf{q}_{\mathrm{L}}^{s} \\
\mathbf{f}_{\mathrm{R}}^{s}=-\lambda_{\mathrm{x}} \mathbf{f}_{\mathrm{L}}^{s}
\end{gathered}
$$

Substituting Eqs. (17) and (20) in Eq. (18), the free wave propagation is described by the eigenproblem

$$
\lambda_{x}\left\{\begin{array}{c}
\mathbf{q}_{\mathrm{L}}^{s} \\
\mathbf{f}_{\mathrm{L}}^{s}
\end{array}\right\}=\mathbf{T}\left\{\begin{array}{c}
\mathbf{q}_{\mathrm{L}}^{s} \\
\mathbf{f}_{\mathrm{L}}^{s}
\end{array}\right\}
$$

whose eigenvalues $\lambda_{x}\left(\omega, k_{y}\right)$ and eigenvectors $\phi\left(\omega, k_{y}\right)=\left[\begin{array}{c}\phi_{q} \\ \phi_{f}\end{array}\right]_{[2 j \times 2 j]} \quad$ solution sets provide a comprehensive description of the propagation constants and the wave mode shapes for each of the elastic waves propagating in the structural waveguide at a specified circular frequency, $\omega$ and wavenumber along $y, k_{y}$. Both positive going (with $\lambda_{x}^{\text {inc }}$ and $\phi^{\text {inc }}$ ) and negative going (with $\lambda_{x}^{\text {ref }}$ and $\boldsymbol{\phi}^{\text {ref }}$ ) waves are sought through the eigensolution. Positive going waves are characterised [15] by

$$
\begin{gathered}
\left|\lambda_{x}^{\mathrm{inc}}\right| \leq 1, \\
\mathfrak{R}\left(\mathrm{i} \omega \boldsymbol{\phi}_{f}^{\mathrm{inc} \top} \boldsymbol{\phi}_{q}^{\mathrm{inc}}\right)<0, \text { if }\left|\lambda_{x}^{\mathrm{inc}}\right|=1
\end{gathered}
$$

which states that when a wave is propagating in the positive $x$ direction, its amplitude should be decreasing, or that if its amplitude is constant (in the case of propagating waves with no attenuation), then there is time average power transmission in the positive direction. Then the wavenumbers of the waves (at a specified circular frequency and $k_{y}$ ) in the positive $k_{x}^{\text {inc }}$ and the negative $k_{x}^{\text {ref }}$ directions can be determined from the propagation constants as

$$
\begin{aligned}
& k_{x}^{\mathrm{inc}}\left(\omega, k_{y}\right)=-\frac{\ln \left(\lambda_{x}^{\mathrm{inc}}\right)}{\mathrm{iL}_{x}} \\
& k_{x}^{\mathrm{ref}}\left(\omega, k_{y}\right)=-\frac{\ln \left(\lambda_{x}^{\mathrm{ref}}\right)}{\mathrm{iL}_{x}}
\end{aligned}
$$

\section{Finite element modelling of structural damage}

Wave interaction with structural damage in layered structures is considered in this study. This section presents the finite element methodologies for some common damage in composite struc- 
tures. Composite structures are subject to a number of damage types which range from microscopic fibre faults to large, gross impact damage. Among these failure modes, notch, crack, delamination and fibre breakage are common failure modes found in composites $[34,35]$.

FE methods are used to describe the effect of damage on the mechanical behaviour of the coupling joint. This is implemented in the FE model of the coupling joint. Some of these methods include stiffness reduction, element deletion, duplicate node and kinematics based methods. Descriptions of each of these methods and their applicability are given in the following sections.

\subsection{Stiffness reduction method}

It is a known fact that structural defects contribute to a reduction in the overall stiffness properties of the structural segment. In this method, the stiffness loss is incorporated in the FE modelling of the structural segment by multiplying the material property of the structure by a reduction factor $\beta$ as

$$
P=\beta P_{0}, \quad 0<\beta \leq 1
$$

where $P$ is the reduced material property, $P_{0}$ the original magnitude of the property (which can be elastic modulus, shear modulus or density). $\beta$ being the reduction factor, equals unity for a pristine structure. This method is applicable to model cracks and delamination, but it is limited to wave interaction problem where mode conversion is not expected.

\subsection{Element deletion method}

This method is mainly applicable for modelling notches such as holes (fibre fractures) and rectangular notches in composites. Here, an element or a number of elements along the axis of the defect is/are deleted from the structure to simulate the effect of the defect. This leads to a reduction in the overall mass and stiffness of the structure. It is one of the simplest FE damage modelling methods as it doesn't require mesh modification.

\subsection{Node duplication method}

The node duplication method is applicable for modelling various damage types such as single and multiple delamination and cracks, and fibre breakages. 
In this method, nodes along axis of damage, within the structural segment, are disconnected by adding duplicate nodes, which have the same nodal coordinates but different nodes numbers, to the nodes being disconnected. Each duplicate node is assigned to an adjacent element such that when a tensile force is applied, the nodes along the damage front are separated. In this respect, if the original nodes are connected to the left side elements, the duplicate nodes will be connected to the elements on the right side.

An illustration of this method is presented for a structural segment with a through-width damage as presented in Appendix A.

\subsection{Kinematics based method}

This approach has a lot of similarities to the node duplication method. It involves enforcing kinematics to the nodes surrounding damage. The structural segment is segmented into multiple domains along damage front. The stiffness and mass matrices of each domain are generated and coupled to obtain the overall matrices of the structural segment. More details on the approach can be found in [36]. The method is applicable to model delamination, cracks and fibre breakages.

\section{Wave interaction with structural damage in 2D layered structures}

A system of $N$ healthy waveguides connected through a structural coupling joint, which exhibit arbitrarily complex mechanical behaviour such as localised damage, is hereby considered (Fig. 2). The waveguides and the joint are arbitrarily complex and periodic. The coupling joint is fully FE modelled while the propagation constants for the elastic waves travelling within the waveguides are sought through the PST-FE scheme as presented in Section 2.

[Figure 2 about here.]

As earlier stated, each waveguide can be different and hence can support a different number of propagating waves, $W$, at a given frequency, $\omega$ and $k_{y}$. However the cross-sections at the interface of each waveguide and the coupling joint must be similar as shown in Fig. 2. 


\subsection{PST formulation of the coupling joint's equation of motion}

Consider a typical coupling joint along which a wave propagates in the $x$ and $y$ directions. The PST formulation of the joint's fully FE-modelled segment is presented in Fig. 3.

[Figure 3 about here.]

The equation of motion for the fully FE-modelled coupling joint can be written as

$$
M \ddot{\mathbf{z}}+\mathbb{C} \dot{\mathbf{z}}+\mathbb{K} \mathbf{z}=\mathbf{f}^{\mathbf{e}}
$$

where $\mathbb{K}, \mathbb{C}$ and $M$ are stiffness, damping and mass matrices of the coupling joint. $\mathbf{z}$ is the physical displacement vector of the coupling joint and $\mathbf{f}^{\mathbf{e}}$ is the set of elastic forces applied to the coupling joint at its interface with connected waveguides.

As in the case of the waveguides, propagation constants are injected along the $y$ axis and propagation characteristics are sought along the $x$ direction. Hence, displacements and forces vectors are expressed as function of the displacements and forces at a chosen reference edge along the axis of wave injection. As demonstrated in Sec. 2.2, the edge with indices LB, IB and RB is used as the reference edge. The segment's displacements are expressed as

$$
\begin{array}{ccc}
\mathbf{z}_{\mathrm{LT}}=\mathrm{e}^{-\mathrm{i} \mu_{y}} \mathbf{Z}_{\mathrm{LB}}, & \mathbf{Z}_{\mathrm{IT}}=\mathrm{e}^{-\mathrm{i} \mu_{y}} \mathbf{Z}_{\mathrm{IB}}, & \mathbf{Z}_{\mathrm{RT}}=\mathrm{e}^{-\mathrm{i} \mu_{y}} \mathbf{Z}_{\mathrm{RB}} \\
\mathbf{Z}_{\mathrm{LI} 1}=\mathrm{e}^{-\mathrm{i} \frac{2}{3} \mu_{y}} \mathbf{Z}_{\mathrm{LB}}, & \mathbf{Z}_{\mathrm{I} 1}=\mathrm{e}^{-\mathrm{i} \frac{2}{3} \mu_{y}} \mathbf{Z}_{\mathrm{IB}}, & \mathbf{z}_{\mathrm{R} 11}=\mathrm{e}^{-\mathrm{i} \frac{2}{3} \mu_{y}} \mathbf{Z}_{\mathrm{RB}} \\
\mathbf{Z}_{\mathrm{LI} 2}=\mathrm{e}^{-\mathrm{i} \frac{1}{3} \mu_{y}} \mathbf{Z}_{\mathrm{LB}}, & \mathbf{z}_{\mathrm{I} 2}=\mathrm{e}^{-\mathrm{i} \frac{1}{3} \mu_{y}} \mathbf{Z}_{\mathrm{IB}}, & \mathbf{z}_{\mathrm{R} 2}=\mathrm{e}^{-\mathrm{i} \frac{1}{3} \mu_{y}} \mathbf{Z}_{\mathrm{RB}}
\end{array}
$$

and forces expressed as

$$
\begin{aligned}
& \mathbf{f}_{\mathrm{LT}}^{\mathbf{e}}=\mathrm{e}^{-\mathrm{i} \mu_{y}} \mathbf{f}_{\mathrm{LB}}^{\mathbf{e}}, \quad \mathbf{f}_{\mathrm{IT}}^{\mathbf{e}}=\mathrm{e}^{-\mathrm{i} \mu_{y}} \mathbf{f}_{\mathrm{IB}}^{\mathbf{e}}, \quad \mathbf{f}_{\mathrm{RT}}^{\mathbf{e}}=\mathrm{e}^{-\mathrm{i} \mu_{y}} \mathbf{f}_{\mathrm{RB}}^{\mathbf{e}} \\
& \mathbf{f}_{\mathrm{L} I 1}^{\mathbf{e}}=\mathrm{e}^{-\mathrm{i} \frac{2}{3} \mu_{y}} \mathbf{f}_{\mathrm{LB}}^{\mathbf{e}}, \quad \mathbf{f}_{\mathrm{I} 1}^{\mathbf{e}}=\mathrm{e}^{-\mathrm{i} \frac{2}{3} \mu_{y}} \mathbf{f}_{\mathrm{IB}}^{\mathbf{e}}, \quad \mathbf{f}_{\mathrm{RI} 1}^{\mathbf{e}}=\mathrm{e}^{-\mathrm{i} \frac{2}{3} \mu_{y}} \mathbf{f}_{\mathrm{RB}}^{\mathbf{e}} \\
& \mathbf{f}_{\mathrm{L} 22}^{\mathbf{e}}=\mathrm{e}^{-\mathrm{i} \frac{1}{3} \mu_{y}} \mathbf{f}_{\mathrm{LB}}^{\mathbf{e}}, \quad \mathbf{f}_{\mathrm{I} 2}^{\mathbf{e}}=\mathrm{e}^{-\mathrm{i} \frac{1}{3} \mu_{y}} \mathbf{f}_{\mathrm{IB}}^{\mathbf{e}}, \quad \mathbf{f}_{\mathrm{R} 22}^{\mathbf{e}}=\mathrm{e}^{-\mathrm{i} \frac{1}{3} \mu_{y}} \mathbf{f}_{\mathrm{RB}}^{\mathbf{e}}
\end{aligned}
$$

with

$$
\mu_{y}=k_{y} L_{J y}
$$


Expressing Eqs. (26) and (27) in tensorial forms gives

$$
\begin{aligned}
& \mathbf{z}=\mathbb{R}_{\mathrm{T}}\left\{\begin{array}{lll}
\mathbf{z}_{\mathrm{LB}} & \mathbf{z}_{\mathrm{IB}} & \mathbf{z}_{\mathrm{RB}}
\end{array}\right\}^{\top} \\
& \mathbb{R}_{\mathrm{T}}^{*} \mathbf{f}^{\mathbf{e}}=\left\{\begin{array}{lll}
\mathbf{f}_{\mathrm{LB}}^{\mathbf{e}} & \mathbf{f}_{\mathrm{IB}}^{\mathbf{e}} & \mathbf{f}_{\mathrm{RB}}^{\mathbf{e}}
\end{array}\right\}^{\top}
\end{aligned}
$$

where $\mathbb{R}_{\mathrm{T}}$ is the transformation matrix for the displacements and $\mathbb{R}_{\mathrm{T}}^{*}$, which is the Hermitian of $\mathbb{R}_{\mathrm{T}}$, is the transformation matrix for the forces. Generally, for a coupling joint of $n$ periodic layers along the axis of wave injection ( $y$ axis in this case), the transformation matrix based on the predefined sequence (Fig. 3) can be expressed as

$$
\mathbf{R}_{\mathrm{T}}=\left[\begin{array}{ccc}
\gamma_{y}^{\frac{n}{n}} \mathbf{I} & \mathbf{0} & \mathbf{0} \\
\mathbf{0} & \gamma_{y}^{\frac{n}{n}} \mathbf{I} & \mathbf{0} \\
\mathbf{0} & \mathbf{0} & \gamma_{y}^{\frac{n}{n}} \mathbf{I} \\
\gamma_{y}^{\frac{n-1}{n}} \mathbf{I} & \mathbf{0} & \mathbf{0} \\
\mathbf{0} & \gamma_{y}^{\frac{n-1}{n}} \mathbf{I} & \mathbf{0} \\
\mathbf{0} & \mathbf{0} & \gamma_{y}^{\frac{n-1}{n}} \mathbf{I} \\
\gamma_{y}^{\frac{n-2}{n}} \mathbf{I} & \mathbf{0} & \mathbf{0} \\
\mathbf{0} & \gamma_{y}^{\frac{n-2}{n}} \mathbf{I} & \mathbf{0} \\
\mathbf{0} & \mathbf{0} & \gamma_{y}^{\frac{n-2}{n}} \mathbf{I} \\
\vdots & \ddots & \vdots \\
\gamma_{y}^{\frac{0}{n}} \mathbf{I} & \mathbf{0} & \mathbf{0} \\
\mathbf{0} & \gamma_{y}^{\frac{0}{n}} \mathbf{I} & \mathbf{0} \\
\mathbf{0} & \mathbf{0} & \gamma_{y}^{\frac{0}{n}} \mathbf{I}
\end{array}\right]
$$

with

$$
\gamma_{y}=\mathrm{e}^{-\mathrm{i} \mu_{y}}
$$

Substituting Eq. (29) into Eq. (25) gives a reformulated equation of motion for the joint as 


$$
\left[\mathbb{R}_{\mathrm{T}}^{*} \mathbb{K} \mathbb{R}_{\mathrm{T}}+\mathrm{i} \omega \mathbb{R}_{\mathrm{T}}^{*} \mathbb{C R} \mathbb{R}_{\mathrm{T}}-\omega^{2} \mathbb{R}_{\mathrm{T}}^{*} M \mathbb{R}_{\mathrm{T}}\right]\left\{\begin{array}{c}
\mathbf{z}_{\mathrm{LB}} \\
\mathbf{z}_{\mathrm{IB}} \\
\mathbf{z}_{\mathrm{RB}}
\end{array}\right\}=\left\{\begin{array}{c}
\mathbf{f}_{\mathrm{LB}}^{\mathbf{e}_{\mathrm{BB}}} \\
\mathbf{f}_{\mathrm{IB}} \\
\mathbf{f}_{\mathrm{RB}}
\end{array}\right\}
$$

In the reformulated equation of motion, the segment is partitioned with regards to its left $L B$, right $R B$ and internal $I B$ DoF which are denoted as $₫ 1$, $₫ 2$ and $m$ as shown in Fig. 3. The circular frequency $(\omega)$ and $k_{y}$ dependent DSM of the joint can then be expressed as

$$
\mathbb{D}=\mathbb{R}_{\mathrm{T}}^{*} \mathbb{K} \mathbb{R}_{\mathrm{T}}+\mathrm{i} \omega \mathbb{R}_{\mathrm{T}}^{*} \mathbb{C R}_{\mathrm{T}}-\omega^{2} \mathbb{R}_{\mathrm{T}}^{*} M \mathbb{R}_{\mathrm{T}}
$$

It is assumed that all connected waveguides are considered to be purely elastic and that no external force is applied at the non-interface nodes of the joint. As a result of this, the DSM of the joint

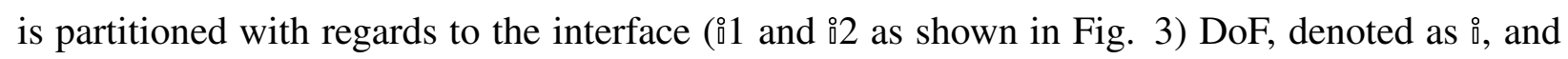
non-interface DoF, denoted as $m$, of the joint with the waveguides as

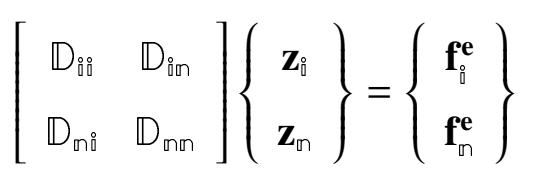

Applying dynamic condensation on the non-interface $\operatorname{DoF}\left(\mathbf{f}_{\mathrm{m}}^{\mathbf{e}}=\mathbf{0}\right)$, Eq. (34) can be expressed as

$$
\mathbb{D}_{\jmath} \mathbf{Z}_{\mathfrak{0}}=\mathbf{f}_{\grave{0}}^{\mathbf{e}}
$$

with

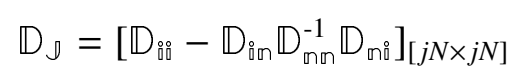

\subsection{Calculation of wave scattering coefficients}

Each supported wavemode $w$ with $w \in[1 \cdots W]$ for waveguide $n$ with $n \in[1 \cdots N]$ in the system (Fig. 2) can be grouped as 


$$
\begin{aligned}
\boldsymbol{\Phi}_{n, q}^{\mathrm{inc}} & =\left[\begin{array}{llll}
\boldsymbol{\phi}_{q, 1}^{\mathrm{inc}} & \boldsymbol{\phi}_{q, 2}^{\mathrm{inc}} & \cdots & \boldsymbol{\phi}_{q, W}^{\mathrm{inc}}
\end{array}\right]_{[j \times W]} \\
\boldsymbol{\Phi}_{n, f}^{\mathrm{inc}} & =\left[\begin{array}{llll}
\boldsymbol{\phi}_{f, 1}^{\mathrm{inc}} & \boldsymbol{\phi}_{f, 2}^{\mathrm{inc}} & \cdots & \boldsymbol{\phi}_{f, W}^{\mathrm{inc}}
\end{array}\right]_{[j \times W]} \\
\boldsymbol{\Phi}_{n, q}^{\mathrm{ref}} & =\left[\begin{array}{llll}
\boldsymbol{\phi}_{q, 1}^{\mathrm{ref}} & \boldsymbol{\phi}_{q, 2}^{\mathrm{ref}} & \cdots & \boldsymbol{\phi}_{q, W}^{\mathrm{ref}}
\end{array}\right]_{[j \times W]} \\
\boldsymbol{\Phi}_{n, f}^{\mathrm{ref}} & =\left[\begin{array}{llll}
\boldsymbol{\phi}_{f, 1}^{\mathrm{ref}} & \boldsymbol{\phi}_{f, 2}^{\mathrm{ref}} & \cdots & \boldsymbol{\phi}_{f, W}^{\mathrm{ref}}
\end{array}\right]_{[j \times W]}
\end{aligned}
$$

The wavemodes of the entire waveguides in the system, at each specified circular frequency, $\omega$ and $k_{y}$, can be grouped as

$$
\boldsymbol{\Phi}_{q}^{\mathrm{inc}}\left(\omega, k_{y}\right)=\left[\begin{array}{cccc}
\boldsymbol{\Phi}_{1, q}^{\mathrm{inc}} & \mathbf{0} & \cdots & \mathbf{0} \\
\mathbf{0} & \boldsymbol{\Phi}_{2, q}^{\mathrm{inc}} & \cdots & \mathbf{0} \\
\cdots & \cdots & \cdots & \cdots \\
\mathbf{0} & \mathbf{0} & \cdots & \boldsymbol{\Phi}_{N, q}^{\mathrm{inc}}
\end{array}\right]_{[j N \times W N]}
$$

with respective expressions for $\boldsymbol{\Phi}_{f}^{\mathrm{inc}}, \boldsymbol{\Phi}_{q}^{\mathrm{ref}}$, and $\boldsymbol{\Phi}_{f}^{\mathrm{ref}}$. For each waveguide, the local coordinate system is defined such that the waveguide's axis is directed towards the joint as shown in Fig. 2. The rotation matrix $\mathbf{R}_{n}$ transforms the DoFs of each waveguide from the local to the global coordinates of the system as

$$
\boldsymbol{\Phi}_{q}^{\mathrm{g}, \text { inc }}=\mathbf{R} \boldsymbol{\Phi}_{q}^{\mathrm{inc}}
$$

with respective similar expressions for $\boldsymbol{\Phi}_{f}^{\mathrm{g}, \text { inc }}, \boldsymbol{\Phi}_{q}^{\mathrm{g}, \text { ref }}$ and $\boldsymbol{\Phi}_{f}^{\mathrm{g} \text {,ref }}$. g denotes the global coordinates index and $\mathbf{R}$ represents the rotation matrices of the system's waveguides, grouped in a block diagonal matrix as

$$
\mathbf{R}=\left[\begin{array}{cccc}
\mathbf{R}_{1} & \mathbf{0} & \cdots & \mathbf{0} \\
\mathbf{0} & \mathbf{R}_{2} & \cdots & \mathbf{0} \\
\cdots & \cdots & \cdots & \cdots \\
\mathbf{0} & \mathbf{0} & \cdots & \mathbf{R}_{N}
\end{array}\right]_{[j N \times j N]}
$$

The equilibrium of forces at the coupling joint interfaces with the waveguides is expressed as 


$$
\mathbf{f}_{0}^{\mathbf{e}}-\mathbf{R f}=0
$$

where $\mathbf{f}$ is the set of forces applied by the waveguides connected to the joint. The continuity conditions for the joint give

$$
\mathbf{z}_{0}=\mathbf{R q}
$$

with

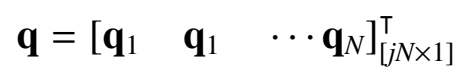

As shown in Fig. 2, waves of amplitudes $\mathbf{a}_{n}^{\text {inc }}$ are impinging on the coupling joint from the $n$th waveguide will give rise to reflected waves of amplitudes $\mathbf{a}_{n}^{\text {ref }}$ in the $n$th waveguide and transmitted waves of amplitudes $\mathbf{a}_{k}^{\text {ref }}$ in the $k$ th waveguide (and vice versa). The reflected and transmitted waves amplitudes are related to the reflection $\mathbf{c}_{n, n}$ and transmission $\mathbf{c}_{k, n}$ coefficients of the scattered waves as

$$
\begin{aligned}
\mathbf{a}_{n}^{\text {ref }} & =\mathbf{c}_{n, n} \mathbf{a}_{n}^{\text {inc }} \\
\mathbf{a}_{k}^{\text {ref }} & =\mathbf{c}_{k, n} \mathbf{a}_{n}^{\text {inc }}
\end{aligned}
$$

Hence, the incident waves amplitudes can be related to the amplitudes of the scattered waves as

$$
\mathbf{a}^{\text {ref }}=\mathbf{S a}^{\text {inc }}
$$

where $\mathbf{a}_{[W N \times l]}^{\text {inc }}$ is the vector containing the amplitudes of the incident waves moving towards the coupling joint and $\mathbf{a}^{\text {ref }}{ }_{[W N \times I]}$ the vector containing the amplitudes of the reflected and transmitted scattered waves. The wave scattering matrix $\mathbf{S}$ whose diagonal and off-diagonal elements respectively represent the reflection and transmission coefficients of the scattered waves can be expressed in the form 


$$
\mathbf{S}=\left[\begin{array}{ccccc}
\mathbf{c}_{1,1} & \cdots & \mathbf{c}_{1, W} & \cdots & \mathbf{c}_{1, W N} \\
\cdots & \cdots & \cdots & \cdots & \cdots \\
\mathbf{c}_{W, 1} & \cdots & \mathbf{c}_{W, W} & \cdots & \mathbf{c}_{W, W N} \\
\cdots & \cdots & \cdots & \cdots & \cdots \\
\mathbf{c}_{W N, 1} & \cdots & \mathbf{c}_{W N, W} & \cdots & \mathbf{c}_{W N, W N}
\end{array}\right]_{[W N \times W N]}
$$

In order to calculate the scattering matrix, an expression is defined for the motion in the waveguides between the physical domain, where the motion is described in terms of $\mathbf{q}$ and $\mathbf{f}$ and the wave domain, where the motion is described in terms of waves of amplitudes $\mathbf{a}^{\text {inc }}$ and $\mathbf{a}^{\text {ref }}$ travelling in the positive and negative directions respectively as

$$
\begin{aligned}
& \mathbf{q}_{n}=\boldsymbol{\Phi}_{n, q}^{\mathrm{inc}} \mathbf{a}_{n}^{\mathrm{inc}}+\boldsymbol{\Phi}_{n, q}^{\mathrm{ref}} \mathbf{a}_{n}^{\text {ref }} \\
& \mathbf{f}_{n}=\boldsymbol{\Phi}_{n, f}^{\mathrm{inc}} \mathbf{a}_{n}^{\mathrm{inc}}+\boldsymbol{\Phi}_{n, f}^{\mathrm{ref}} \mathbf{a}_{n}^{\text {ref }}
\end{aligned}
$$

and by concatenating the corresponding vectors and matrices, the general expressions for $\mathbf{q}$ and $\mathbf{f}$ for the system's waveguides can be expressed as

$$
\begin{aligned}
& \mathbf{q}=\boldsymbol{\Phi}_{q}^{\mathrm{inc}} \mathbf{a}_{n}^{\mathrm{inc}}+\boldsymbol{\Phi}_{q}^{\mathrm{ref}} \mathbf{a}_{n}^{\mathrm{ref}} \\
& \mathbf{f}=\boldsymbol{\Phi}_{f}^{\mathrm{inc}} \mathbf{a}_{n}^{\mathrm{inc}}+\boldsymbol{\Phi}_{f}^{\mathrm{ref}} \mathbf{a}_{n}^{\mathrm{ref}}
\end{aligned}
$$

Then substituting Eq. 35 into the equilibrium equation (Eq. 41) and the continuity equation (Eq. 42) into the resulting expression gives

$$
\mathbb{D}_{\jmath} \mathbf{R q}=\mathbf{R f}
$$

Substituting Eqs. 39 and 48 in Eq. 49 and express the resulting equation in the form of Eq. 45 gives the wave interaction scattering matrix (at a specified circular frequency, $\omega$, and imposed wave constant along $y, k_{y}$ ) as

$$
\boldsymbol{S}\left(\omega, k_{y}\right)=-\left[\boldsymbol{\Phi}_{f}^{\mathrm{g}, \text { ref }}-\mathbb{D}_{\downarrow} \boldsymbol{\Phi}_{q}^{\mathrm{g}, \mathrm{ref}}\right]^{-1}\left[\boldsymbol{\Phi}_{f}^{\mathrm{g}, \text { inc }}-\mathbb{D}_{\downarrow} \boldsymbol{\Phi}_{q}^{\mathrm{g}, \text {,inc }}\right]
$$




\section{Numerical case studies}

This section presents numerical case studies to demonstrate the validity and applicability of the developed methodology. The case examples are divided into two parts, namely validation and test case studies. In the first part, structural model whose wave dispersion and scattering properties can easily be obtained theoretically is presented. The theoretical results are compared to the numerical results obtained through the presented scheme in order to demonstrate the validity of the scheme. The second part, test case studies, presents the application of the scheme to quantify wave interaction with defects in damaged layered structures, and characterise damage types based on calculated wave interaction coefficients.

In all the example cases, FE mesh size of a structural segment is chosen based on the theoretical dispersion curves for the segment. This is to ensure that the mesh density is fine enough to represent the structure accurately at a reasonable computational time. All properties and dimensions are in SI units, unless otherwise stated.

\subsection{Validation case studies}

The first illustrative example of the presented computational scheme is a cracked aluminium beam system (Fig. 8). The configuration comprises two healthy waveguides connected through a coupling joint within which crack is implemented. The three waveguides in the system can in general have different material characteristics however in this case they are assumed to be made of aluminium with material properties $E=70 \times 10^{9} \mathrm{~Pa}, \rho=2600 \mathrm{~kg} / \mathrm{m}^{3}$ and $v=0.3$. The entire beam has a uniform cross section with $L_{1}=L_{2}=0.2 \mathrm{~m}, L_{J}=0.05 \mathrm{~m}, b=0.001 \mathrm{~m}$ and $h=0.003$ m.

The Euler-Bernoulli beam supports longitudinal, torsional and bending waves whose respective wavenumbers $k_{l}, k_{t}$ and $k_{b}$ can be obtained analytically as 


$$
\begin{gathered}
k_{l}=\omega \sqrt{\frac{\rho\left(1-v^{2}\right)}{E}} \\
k_{t}=\omega \sqrt{\frac{\rho}{G}} \\
k_{b}=\sqrt[4]{\frac{\omega^{2} \rho h}{D}}
\end{gathered}
$$

where $G=E / 2(1+v)$ is the shear modulus and $D=E h^{3} / 12\left(1-v^{2}\right)$ is the bending stiffness of the beam.

Since $k_{x}=k \cos \theta, k_{y}=k \sin \theta$ for a wave travelling at an angle $\theta$ to x-direction, and hence, $k^{2}=k_{x}^{2}+k_{y}^{2}$, the analytical wavenumber of the wave along $\mathrm{x}$ and $\mathrm{y}$ directions are defined as

$$
\begin{aligned}
& k_{x}^{2}=k^{2}-k_{y}^{2} \\
& k_{y}^{2}=k^{2}-k_{x}^{2}
\end{aligned}
$$

A segment of each waveguide is modelled using SOLID185 finite element of length $\Delta=0.001$ in ANSYS. The coupling joint is modelled using similar segment length as the waveguides. Crack within the coupling joint is modelled using the stiffness reduction method with reduction factors ranging from $\beta=0.01$ to 0.09 . This corresponds to damage severity ratio ranging from 0.1 to 0.9 respectively.

In order to validate the presented approach, the results derived through Eq. (21) for the wavenumbers and through Eq. (50) for the wave reflection and transmission coefficients are compared against the analytical results. Analytical wave reflection and transmission coefficients for this particular problem can be obtained as in $[37,38]$.

[Figure 4 about here.]

[Figure 5 about here.]

Results for the dispersion curves using both approaches are presented in Figs. 4 and 5. Excellent agreement is observed, which establishes the validity of the scheme. Fig. 4 presents dispersion curves in the $\left(\omega, k_{x}\right)$ plane for different imposed wave values. For imposed wave $k_{y}=0$, every wave cuts on at circular frequency $\omega=0$ as expected. This is synonymous to one dimensional wave 
propagation with waves propagating only in the $\mathrm{x}$-direction. Cut on frequencies of the waves are observed to increase as $k_{y}$ value increases. Fig. 5 presents dispersion curves in the $\left(k_{x}, k_{y}\right)$ plane for different circular frequency values. As observed, the contour curves are independent of propagation direction. This is due to the isotropic nature of the beam under consideration, in which it is expected that material properties will be independent of direction.

[Figure 6 about here.]

[Figure 7 about here.]

Figs. 6 and 7 present the reflection and transmission efficiencies for the three wave modes present in the beam. Excellent agreement is observed between the analytical results $[37,38]$ and that of the present scheme. As a conservation of energy, the reflection and transmission efficiencies of a wave propagating through an undamped system should sum up to unity. This is observed for both sets of results presented in Fig. 6. Fig. 7 presents the dependence of wave reflection coefficients on damage severity for each wave mode. It is observed that the coefficient magnitude increases as damage becomes more severe.

\subsection{Test case studies}

\subsubsection{Orthotropic beam}

An arbitrarily layered orthotropic beam having a uniform cross-sectional area $(b=0.01 \mathrm{~m}$ and $h=0.01 \mathrm{~m})$ is considered. The beam is defined as a system of two pristine beams $\left(L_{1}=L_{2}=0.2\right.$ m) connected through a damaged beam $\left(L_{J}=0.01 \mathrm{~m}\right)$ as shown in Fig. 8. Each beam comprises ten layers of glass-epoxy composite material whose material properties are $E_{x}=45.6 \times 10^{9} \mathrm{~Pa}$, $E_{y}=E_{z}=16.2 \times 10^{9} \mathrm{~Pa}, G_{x y}=G_{x z}=5.83 \times 10^{9} \mathrm{~Pa}, G_{y z}=5.79 \times 10^{9} \mathrm{~Pa}, v_{x y}=v_{x z}=0.278$, $v_{y z}=0.4$ and $\rho=2000 \mathrm{~kg} / \mathrm{m}^{3}$.

[Figure 8 about here.]

A segment (of length $\Delta=0.001 \mathrm{~m}$ ) of each waveguide is modelled in ANSYS with 100 SOLID185 finite elements using cubed sized elements of length $0.001 \mathrm{~m}$. Dispersion curves for the waveguides are obtained by solving Eq. (21) within frequency range $\omega=\left(1.0 \times 10^{2}-6.0 \times 10^{4}\right) \mathrm{Hz}$. 
Three propagating modes are obtained within the frequency range. The dispersion curves for these waves in the $\left(\omega, k_{x}\right)$ and $\left(k_{y}, k_{x}\right)$ axes, for $k_{y}=10 \mathrm{~m}^{-1}$ and $\omega=50 \mathrm{kHz}$ respectively, are presented in Fig. 9.

[Figure 9 about here.]

As expected, for an orthotropic beam, the dispersion property of the beam depends on the propagation direction of the waves. As shown in Fig. 9, it is observed that the cut-on frequency of a wave propagating in the $\mathrm{x}$ direction greatly depends on the imposed wavenumber in the y direction, $k_{y}$. The higher the imposed $k_{y}$, the higher the cut-on frequencies of the propagating waves. It is also observed that at a given frequency, the wavenumbers of the waves propagating along $\mathrm{x}$ direction reduce with respect to $k_{y}$.

Wave interaction properties of the beam for different failure modes within the coupling joint of the beam are studied in the next sections. Dependence of the waves reflection coefficients on the severities of the failure modes are analysed in order to establish optimal parameters for detecting and identifying damage types analysed.

In each case, the coupling joint is modelled using similar element size as the waveguides. Hence, the coupling joint is modelled with 1000 SOLID185 finite elements. Damage parameters depend on damage type being analysed as presented in next sections. In each case, Eq. (50) is solved for the wave interaction coefficients from the damaged coupling joint. Generally, notches are modelled using the element deletion method while cracks and delaminations are modelled using the node duplication approach.

\subsubsection{Dependence of wave interaction coefficient on crack within the orthotropic beam}

In this case, wave reflection from zero-width crack within the beam's coupling joint, as shown in Fig. 8, is considered. The crack is situated at mid length of the joint, and runs transversely through the breadth of the joint. In order to analyse the dependence of the wave reflection coefficient on the severity of crack, several crack depths are considered. Crack depths within the range $(0.001$ $0.009) \mathrm{m}$, which correspond to ratios $(0.10 .9)$ of the beam's thickness, are analysed. 
[Figure 10 about here.]

[Figure 11 about here.]

Fig. 10 shows the variations of the bending wave reflection coefficient with frequency as well as with $k_{y}$ for different crack depths. It is observed that the nature of frequency and $k_{y}$ dependence of the reflection coefficient is closely similar for the various crack depths. With respect to frequency, the reflection coefficient have a minimum, beyond which the reflection coefficient increases with frequency and before which it decreases. The frequency bandwidth, at which the reflection coefficient reaches a minimum increases inversely with crack depth. That is, lower depth crack will exhibit wider bandwidth. On the other hand, the reflection coefficient changes very slightly with respect to $k_{y}$ for the various crack depths. This trend is analogous to that obtained for the bending wave's dispersion curve $k_{y}$ versus $k_{x}$ as shown in Fig. 9.

Fig. 11 presents the longitudinal reflection coefficient as a function of frequency and $k_{y}$ at various crack depths. In relation to frequency, the reflection coefficient magnitude increases steadily, though not linear especially for deeper crack, until it becomes plateaued. On the other hand, with respect to $k_{y}$, the reflection magnitude decreases very slightly over low to mid $k_{y}$ range, then reduces rapidly towards zero. This is also in similar fashion to the trend obtained for the longitudinal wavenumber against $k_{y}(9)$.

\subsubsection{Dependence of wave interaction coefficient on notch within the orthotropic beam}

In this case, wave reflection from $1 \mathrm{~mm}$ wide notch within the beam's coupling joint, as shown in Fig. 8, is considered. The notch is situated at mid length of the joint, and runs transversely through the breadth of the joint. In order to analyse the dependence of the wave reflection coefficient on the severity of notch, notch depths, within the range $(0.0010 .009) \mathrm{m}$, which correspond to ratios (0.1 0.9) of the beam's thickness are analysed.

[Figure 12 about here.]

[Figure 13 about here.] 
Reflection coefficient results with respect to frequency as well as $k_{y}$ for different notch depths are presented in Figs. 12 and 13. With respect to frequency, the reflection coefficient of the bending wave has a minimum, beyond which the reflection coefficient increases with frequency and before which it decreases. The frequency at which the reflection coefficient reaches a minimum increases inversely with crack depth. Reflection coefficient of the longitudinal wave increases steadily until it becomes plateaued. With respect to $k_{y}$, reflection coefficient of the bending wave changes very slightly with respect to $k_{y}$ for the various crack depths while that of the longitudinal wave decreases very slightly over low to mid $k_{y}$ range, then reduces rapidly towards zero.

5.2.1.3 Dependence of wave interaction coefficient on delamination within the orthotropic beam Wave reflection from interlaminar delamination within the beam's coupling joint (Fig. 8) is considered in this case. The delamination is situated at mid layer (between the fifth and sixth plies) of the joint, and runs transversely through the breadth of the joint. Delamination widths in the range (0.001 0.009$) \mathrm{m}$ are considered. These correspond to ratios $\left(\begin{array}{ll}0.1 & 0.9\end{array}\right)$ of the beam's thickness.

[Figure 14 about here.]

[Figure 15 about here.]

Reflection coefficient results with respect to circular frequency as well as $k_{y}$ for different notch depths are presented in Figs. 14 and 15. With respect to circular frequency, reflection coefficient of the bending wave increases until it becomes plateaued. Reflection coefficient of the longitudinal wave also increases with circular frequency, but only slightly. With respect to $k_{y}$, reflection coefficient of the bending wave decreases slightly over low to mid $k_{y}$ range, then, beyond this range, reduces rapidly. Reflection coefficient of the longitudinal wave increases very slightly until high $k_{y}$ range, then increases more significantly.

5.2.1.4 Comparison of wave interaction coefficient for the different damage types within the orthotropic beam

Having computed wave interaction characteristics for different damage types in the orthotropic beam, it is essential to evaluate the sensitivity of each damage type to wave reflection in order 
to establish optimal parameter for detecting and identifying damage type based on their wave interaction properties.

[Figure 16 about here.]

[Figure 17 about here.]

Figs. 16 and 17 present wave reflection coefficient against damage severity for the various damage types considered above. As observed, crack and notch are more sensitive to reflection compared to delamination. However, the sensitivity of delamination to bending wave reflection is higher compared to that of longitudinal wave reflection. Though, reflection coefficient from crack and notch are both highly significant for the two wave modes, it can be observed that reflection from both damage types are observed to be more significant for the longitudinal wave compared to the bending wave. Also, at each damage severity, reflection from crack is higher than that of notch by about $5 \%$.

From these observations, it therefore follows that bending wave is the proper wave mode to detect interlaminar delamination within the orthotropic beam while either bending or longitudinal is good for detecting crack and notch within the beam though longitudinal mode is more recommended. Applying the trend equations indicated on the Figures, for a particular damage severity, applicable damage type can be identified using the wave interaction coefficient.

\subsubsection{Sandwich laminate}

A sandwich laminate, made of a foam core sandwiched between two skin laminae, is considered. The upper and lower skin laminae each consists of one orthotropic layer of glass-epoxy of material properties: $\rho=2000 \mathrm{~kg} / \mathrm{m}^{3}, E_{x}=E_{y}=54 \times 10^{9} \mathrm{~Pa}, E_{z}=48 \times 10^{9} \mathrm{~Pa}, G_{x y}=3.16 \times 10^{9}$ $\mathrm{Pa}, G_{y z}=G_{x z}=1.78 \times 10^{9} \mathrm{~Pa}, v_{x y}=0.06, v_{y z}=v_{x z}=0.313, h_{s 1}=h_{s 2}=0.001 \mathrm{~m}$. The foam core consists of eight layers of honeycomb material of properties: $E=18 \times 10^{7} \mathrm{~Pa}, \rho=110 \mathrm{~kg} / \mathrm{m}^{3}$, $v=0.28, h_{c}=0.008 \mathrm{~m}$.

[Figure 18 about here.] 
The laminate system is discretised as two healthy waveguides $\left(L_{1}=L_{2}=0.2 \mathrm{~m}\right)$ coupled through a damaged joint $\left(L_{J}=0.01 \mathrm{~m}\right)$ as shown in Fig. 18. The laminate's cross-section $(b=0.01 \mathrm{~m}$, $h=0.01 \mathrm{~m})$ is constant throughout and are fixed at both ends.

A segment (of length $\Delta=0.001 \mathrm{~m}$ ) of each waveguide is modelled in ANSYS with 100 SOLID185 finite elements (10 for each skin and 80 for the core) using cubed sized elements of length $0.001 \mathrm{~m}$. Dispersion curves for the waveguides are obtained by solving Eq. (21) within frequency range $\omega=\left(1.0 \times 10^{2}-6.0 \times 10^{4}\right) \mathrm{Hz}$. Three propagating modes are obtained within the frequency range. The dispersion curves for these waves in the $\left(\omega, k_{x}\right)$ and $\left(k_{y}, k_{x}\right)$ axes, for $k_{y}=2$ $\mathrm{m}^{-1}$ and $\omega=30 \mathrm{kHz}$ respectively, are presented in Fig. 19.

[Figure 19 about here.]

As in the orthotropic beam, the dispersion property of the sandwich laminate depends on the propagation direction of the waves. As shown in Fig. 9, it is observed that the cut-on frequency of a wave propagating in the $\mathrm{x}$ direction greatly depends on the imposed wavenumber in the $\mathrm{y}$ direction, $k_{y}$. The higher the imposed $k_{y}$, the higher the cut-on frequencies of the waves. It is also observed that at a given frequency, the wavenumbers of the waves propagating along $\mathrm{x}$ direction reduce with respect to $k_{y}$.

Wave reflection coefficient from the coupling joint, with different damage types, are calculated using Eq. (50). Dependence of the reflection properties on the severities of the failure modes are analysed in order to establish basis for detecting and identifying a specific damage type based on its wave reflection characteristics.

In each damage type case, the coupling joint is modelled with similar element size used for the waveguides. Hence, the joint consists of 1000 SOLID185 finite elements. As presented in next sections, damage parameters depends on damage type being analysed. Generally, notches are modelled using the element deletion method while cracks and delaminations are modelled using the node duplication approach. 


\subsubsection{Dependence of wave interaction coefficient on crack within the sandwich laminate}

In this case, wave reflection from zero-width crack within the laminate's coupling joint, as shown in Fig. 18, is considered. The crack is situated at mid length of the joint, and runs transversely through the breadth of the joint. In order to analyse the dependence of the wave reflection coefficient on the severity of crack, several crack depths are considered. Crack depths within the range (0.001 - 0.009) m, which correspond to ratios (0.1 - 0.9) of the laminate's thickness, are analysed. Variations of the bending and longitudinal waves reflection coefficients with frequency as well as with $k_{y}$ for different crack depths are presented in Figs. 20 and 21.

[Figure 20 about here.]

[Figure 21 about here.]

With respect to circular frequency, the bending wave reflection coefficient exhibits a sinusoidaltype of relationship. The reflection coefficient increases to a maximum then reduces to a minimum, then increases back to a maximum and so on. Generally, the reflection coefficient, at a given frequency, increases linearly with crack severity. The longitudinal wave reflection coefficient generally increases with respect to circular frequency. And at a given frequency, it directly increases with regards to crack severity.

In relation to $k_{y}$, the bending wave reflection coefficient changes very slightly until a considerably higher value of $k_{y}$. At a given value of $k_{y}$, the reflection coefficient varies directly with the severity of crack. Similar variation is observed for the longitudinal wave reflection coefficient. However, at a given value of $k_{y}$, change in the wave reflection magnitude, per 0.1 change in crack severity ratio, is more uniform in the longitudinal wave compared to the bending wave. For the longitudinal wave, a uniform average change of about 0.1 is observed. While an average change of about 0.005 and 0.02 , are observed for the bending wave at low and high severity range respectively. 
5.2.2.2 Dependence of wave interaction coefficient on notch within the sandwich laminate In this case, wave reflection from $1 \mathrm{~mm}$ wide notch within the laminate's coupling joint, as shown in Fig. 18, is considered. The notch is situated at mid length of the joint, and runs transversely through the breadth of the joint. In order to analyse the dependence of the wave reflection coefficient on the severity of notch, notch depths, within the range $(0.0010 .009) \mathrm{m}$, which correspond to ratios $(0.10 .9)$ of the laminate's thickness are analysed. Variations of the bending and longitudinal waves reflection coefficients with frequency as well as with $k_{y}$ for different notch severity ratios are presented in Figs. 22 and 23. Results obtained for wave reflection from notch within the laminate follow similar trend as those obtained for wave reflection from crack.

[Figure 22 about here.]

[Figure 23 about here.]

As a function of frequency, bending wave reflection from notch exhibits a sinusoidal-type of relationship while longitudinal wave reflection generally increases as frequency increases. At a given circular frequency, the bending wave reflection coefficient varies directly with notch severity. Similar trend is observed for the longitudinal wave reflection. With respect to $k_{y}$, reflection coefficient magnitudes of the waves change very slightly until a considerably higher value of $k_{y}$. At a given

value of $k_{y}$, the reflection coefficient of both wave modes varies directly with the severity of notch. However, there is an average of 0.1 increase in the longitudinal wave reflection coefficient per 0.1 change of notch severity ratio. Changes in the bending wave reflection coefficient per 0.1 change of notch severity ratio is not uniform; an average change of about 0.02 is observed at low severity ratio while an average change of about 0.25 is observed at higher severity ratio.

5.2.2.3 Dependence of wave interaction coefficient on skin-core interlaminar delamination within the sandwich laminate

Wave reflection from skin-core interlaminar delamination within the laminate's coupling joint, as shown in Fig. 18, is considered in this case. The delamination, situated at the skin-core interface, 
runs transversely through the breadth of the joint. In order to analyse the dependence of the wave reflection coefficient on the severity of delamination, several lengths are considered. Delamination lengths within the range $(0.001-0.009) \mathrm{m}$, which correspond to ratios $(0.1-0.9)$ of the laminate's thickness, are analysed. Variations of the bending and longitudinal waves reflection coefficients with frequency as well as with $k_{y}$ for different skin-core delamination severities are presented in Figs. 24 and 25.

[Figure 24 about here.]

[Figure 25 about here.]

With respect to circular frequency, reflection coefficient of the bending wave increases as frequency increases. Reflection coefficient of the longitudinal wave also increases with circular frequency, but only slightly. With respect to $k_{y}$, reflection coefficient of the bending wave changes very slightly over the considered $k_{y}$ range. Reflection coefficient of the longitudinal wave increases very slightly until high $k_{y}$ range, then increases more significantly. Generally, reflection coefficient of the waves increases with increase in delamination severity ratio.

5.2.2.4 Dependence of wave interaction coefficient on core interlaminar delamination within the sandwich laminate

Wave reflection from core interlaminar delamination within the laminate's coupling joint, as shown in Fig. 18, is considered in this case. The delamination, situated at the mid-height of the joint, runs transversely through the breadth of the joint. In order to analyse the dependence of the wave reflection coefficient on the severity of delamination, several lengths are considered. Delamination lengths within the range $(0.001-0.009) \mathrm{m}$, which correspond to ratios $(0.1-0.9)$ of the laminate's thickness, are analysed. Variations of the bending and longitudinal waves reflection coefficients with frequency as well as with $k_{y}$ for different core delamination severities are presented in Figs. 26 and 27.

[Figure 26 about here.] 
[Figure 27 about here.]

Results obtained follow similar trend as the skin-core delamination. However, the reflection coefficient magnitude are higher in this case.

5.2.2.5 Comparison of wave interaction coefficient dependence on different damage types within the sandwich laminate

The sensitivity of each damage type to wave reflection in the sandwich laminate, in order to establish optimal parameter for detecting and identifying damage type based on their wave interaction properties, is evaluated hereby.

[Figure 28 about here.]

[Figure 29 about here.]

Figs. 28 and 29 present wave reflection coefficient against damage severity for the various damage types within the sandwich laminate. As observed, crack is much more sensitive to longitudinal wave reflection compared to bending wave reflection with an average difference of about $72 \%$. Notch is highly sensitive to both wave modes, but more sensitive to longitudinal wave with an average difference of about $19 \%$. Compared to crack and notch, low reflections are generally observed for the two delamination modes considered. However, the sensitivity of delaminations to bending wave reflection is much more significant compared to that of longitudinal wave.

From these observations, it therefore follows that bending wave is recommended for detecting interlaminar delaminations within the sandwich laminate while either bending or longitudinal mode is good for detecting crack and notch within the laminate though longitudinal mode is more recommended. Based on this recommendation and that made for the orthotropic beam, general recommendation can be reached for horizontally and vertically oriented damage within composite structural media.

Outcome of this analysis is also applicable for predicting and identifying specific damage type within the structural media using the wave interaction coefficient trend equations. 


\section{Concluding remarks}

An FE-based methodology for numerical quantification of wave interaction with localised damage in two-dimensional periodic structures is presented in this study. The presented scheme can be applied to structures of arbitrary layering, complexities and material characteristics due to the FE discretisation being employed. The scheme discretises a damaged structural medium into a system of $N$ healthy substructures connected through a joint which bears the structural damage/discontinuity. Wave propagation constants along different propagation directions of the substructures are sought by coupling Periodic Structure Theory with FE. The damaged joint is modelled using standard FE approach, ensuring joint-substructures meshes conformity. This is coupled with the obtained wave propagation constants in order to compute the scattering coefficients of the wave interaction with damage.

The main outcomes of the work can be summarised as:

(a) The presented scheme is validated against theoretical predictions using a cracked aluminium beam. A very good correlation is observed between the two sets of results.

(b) The scheme is able to quantify wave interaction with localised damage in arbitrarily multilayered two-dimensional structures, which may be difficult to solve using the convectional FE method and at a computational time faster than the conventional FE transient approach.

(c) Wave interaction characteristics for different damage types and parameters are analysed in order to establish an optimal basis for detecting and identifying damage, as well as assessing the orientation and extent of damage.

Further work focusses on extending the scheme to efficient damage identification and structural health diagnosis and prognosis using the presented methodology. This will expand the applicability of the presented scheme.

\section{Data availability}

The raw/processed data required to reproduce these findings cannot be shared at this time as the data also forms part of an ongoing study. They will be made available upon request. 


\section{References}

[1] S. Kessler, S. Spearing, C. Soutis, Damage detection in composite materials using lamb wave methods, Smart materials and structures 11 (2002) 269.

[2] A. Croxford, P. Wilcox, B. Drinkwater, G. Konstantinidis, Strategies for guided-wave structural health monitoring, in: Proceedings of the Royal Society of London A: Mathematical, Physical and Engineering Sciences, 2087, The Royal Society, pp. 2961-2981.

[3] R. Guan, Y. Lu, W. Duan, X. Wang, Guided waves for damage identification in pipeline structures: A review, Structural Control and Health Monitoring 24 (2017) e2007.

[4] E. Manoach, M. Cao, S. Doneva, Vibration based methods for damage detection of plates, in: AIP Conference Proceedings, volume 1922, AIP Publishing, p. 100014.

[5] P. Cawley, R. Adams, The location of defects in structures from measurements of natural frequencies, The Journal of Strain Analysis for Engineering Design 14 (1979) 49-57.

[6] J. Penny, D. Wilson, M. Friswell, Damage location in structures using vibration data, in: PROCEEDINGS-SPIE THE INTERNATIONAL SOCIETY FOR OPTICAL ENGINEERING, SPIE INTERNATIONAL SOCIETY FOR OPTICAL, pp. 861-861.

[7] T. Wolff, M. Richardson, Fault detection in structures from changes in their modal parameters, in: Proceedings of the 7th international modal analysis conference, volume 1, pp. 87-94.

[8] M. Mannan, M. Richardson, Detection and location of structural cracks using frf measurements, 8th IMAC Proceedings, Kissimmee Florida (1990).

[9] P. Mix, Introduction to nondestructive testing: a training guide, John Wiley \& Sons, 2005.

[10] P. Shull, Nondestructive evaluation: theory, techniques, and applications, CRC press, 2016.

[11] D. Alleyne, P. Cawley, Optimisation of lamb wave inspection techniques, NDT\&E International 25 (1992) 11-22.

[12] M. Lowe, O. Diligent, Low-frequency reflection characteristics of the s 0 lamb wave from a rectangular notch in a plate, The Journal of the Acoustical Society of America 111 (2002) 64-74.

[13] M. Lowe, P. Cawley, J. Kao, O. Diligent, The low frequency reflection characteristics of the fundamental antisymmetric lamb wave a 0 from a rectangular notch in a plate, The Journal of the Acoustical Society of America 112 (2002) 2612-2622.

[14] D. Mead, A general theory of harmonic wave propagation in linear periodic systems with multiple coupling, Journal of Sounds and Vibration 27 (1973) 235-260.

[15] B. R. Mace, D. Duhamel, M. J. Brennan, L. Hinke, Finite element prediction of wave motion in structural waveguides, The Journal of the Acoustical Society of America 117 (2005) 2835-2843.

[16] E. Manconi, B. Mace, Modelling wave propagation in two-dimensional structures using a wave/finite element technique, ISVR Technical Memorandum (2007). 
[17] J. Mencik, A wave finite element-based formulation for computing the forced response of structures involving rectangular flat shells, International Journal for Numerical Methods in Engineering 95 (2013) 91-120.

[18] W. Zhou, M. Ichchou, Wave propagation in mechanical waveguide with curved members using wave finite element solution, Computer Methods in Applied Mechanics and Engineering 199 (2010) 2099-2109.

[19] J. Renno, B. Mace, Calculating the forced response of cylinders and cylindrical shells using the wave and finite element method, Journal of Sound and Vibration 333 (2014) 5340-5355.

[20] J. Mencik, M. Ichchou, Multi-mode propagation and diffusion in structures through finite elements, European Journal of Mechanics-A/Solids 24 (2005) 877-898.

[21] D. Duhamel, B. Mace, M. Brennan, Finite element analysis of the vibrations of waveguides and periodic structures, Journal of sound and vibration 294 (2006) 205-220.

[22] J. Renno, B. Mace, On the forced response of waveguides using the wave and finite element method, Journal of Sound and Vibration 329 (2010) 5474-5488.

[23] J. Mencik, On the low-and mid-frequency forced response of elastic structures using wave finite elements with one-dimensional propagation, Computers \& Structures 88 (2010) 674-689.

[24] P. Silva, J. Arruda, Wave spectral finite element analysis of two-dimensional waveguides, Civil-Comp Press Stirlingshire (2012).

[25] W. Zhou, M. Ichchou, J. Mencik, Analysis of wave propagation in cylindrical pipes with local inhomogeneities, Journal of Sound and Vibration 319 (2009) 335-354.

[26] J. Renno, B. Mace, Calculation of reflection and transmission coefficients of joints using a hybrid element/wave and finite element approach, Journal of Sound and Vibration 332 (2013) 2149-2164.

[27] G. Mitrou, N. Ferguson, J. Renno, Wave transmission through two-dimensional structures by the hybrid fe/wfe approach, Journal of Sound and Vibration 389 (2017) 484-501.

[28] M. Ichchou, J. Mencik, W. Zhou, Wave finite elements for low and mid-frequency description of coupled structures with damage, Computer methods in applied mechanics and engineering 198 (2009) 1311-1326.

[29] T. Huang, M. Ichchou, O. Bareille, Multi-mode wave propagation in damaged stiffened panels, Structural Control and Health Monitoring 19 (2012) 609-629.

[30] D. Chronopoulos, M. Ichchou, B. Troclet, O. Bareille, Predicting the broadband vibroacoustic response of systems subject to aeroacoustic loads by a krylov subspace reduction, Applied Acoustics 74 (2013) 1394-1405.

[31] R. Apalowo, D. Chronopoulos, G. Tanner, Wave interaction with defects in pressurised composite structures, Journal of Nondestructive Evaluation 37 (2018) 48.

[32] R. Apalowo, D. Chronopoulos, M. Ichchou, Y. Essa, F. Martin De La Escalera, The impact of temperature on wave interaction with damage in composite structures, Proceedings of the Institution of Mechanical Engineers, Part C: Journal of Mechanical Engineering Science 231 (2017) 3042-3056.

[33] D. Chronopoulos, C. Droz, R. Apalowo, M. Ichchou, W. Yan, Accurate structural identification for layered 
composite structures, through a wave and finite element scheme, Composite Structures 182 (2017) 566-578.

[34] R. Talreja, C. Singh, Damage and Failure of Composite Materials, Damage and Failure of Composite Materials, Cambridge University Press, 2012.

[35] R. Heslehurst, Defects and damage in composite materials and structures, CRC Press, 2014.

[36] A. Nag, D. Mahapatra, S. Gopalakrishnan, T. Sankar, A spectral finite element with embedded delamination for modeling of wave scattering in composite beams, Composites science and Technology 63 (2003) 2187-2200.

[37] J. Doyle, Wave Propagation in Structures: Spectral Analysis Using Fast Discrete Fourier Transforms, Springer, 1997.

[38] L. Cremer, M. Heckel, B. Petersson, Structure Borne Sound: Structural Vibrations and Sound Radiation at Audio Frequencies, Springer, 2005.

[39] ANSYS, ANSYS 14.0 User's Help, 2014.

\section{Appendix A. Illustrative example for the node duplication method}

As an illustration of this method, a structural segment with a through-width damage is considered. The segment is meshed using 80 3D solid finite elements as shown in Fig. 30. Elements and nodes numbering of the segment are as shown in Fig. 30. Nodes and elements arrangements are based on the nodes and elements connectivities for 3D SOLID185 elements [39]. For the damage depth considered, nodes 100, 101, 102, 103, 104, 105, 106, 107 and 108, which are along the damage path, are respectively disconnected by adding duplicate nodes 163, 164, 165, 166, 167, $168,169,170$ and 171 of same respective nodal coordinates.

[Figure 30 about here.]

In a pristine state of the segment, the nodal connectivities of elements $45,46,47$ and 48 are $[73,100,101,74,76,103,104,77],[74,101,102,75,77,104,105,78],[76,103,104,77,79$, $106,107,80]$ and $[77,104,105,78,80,107,108,81]$ respectively. That of elements $61,62,63$ and 64 are respectively [100, 127, 128, 101, 103, 130, 131, 104], [101, 128, 129, 102, 104, 131, 132, 105], [103, 130, 131, 104, 106, 133, 134, 107] and [104, 131, 132, 105, 107, 134, 135, 108]. But, in a damaged state, the nodal connectivities of elements 45, 46, 47 and 48 remain unchanged while that of elements $61,62,63$ and 64 become [163, 127, 128, 164, 166, 130, 131, 167], [164, 128, $129,165,167,131,132,168],[166,130,131,167,169,133,134,170]$ and $[167,131,132,168$, 
$170,134,135,171]$ respectively. In this sense, the duplicates nodes are attributed to the elements on the right side of the damage axis such that when a tensile force is applied, elements $61,62,63$ and 64 will be separated from elements $45,46,47$ and 48 thereby simulating the damage. 


\section{List of Figures}

1 Periodic FE-modelled segment of an arbitrarily layered composite structure . . .

2 Periodic waveguides connected through a coupling joint which carries localised damage. A positive going wave of amplitude $\mathbf{a}_{n}^{\text {inc }}$ impinging on the joint from $n$th waveguide will give rise to wave of reflection coefficient $\mathbf{c}_{n, n}$ in the $n$th waveguide and wave of transmission coefficient $\mathbf{c}_{k, n}$ in the $k$ th waveguide . . . . . . .

3 PST formulation of a typical fully FE-modelled coupling joint . . . . . . . . .

4 Dispersion curves for the beam: bending (3), torsional (2) and longitudinal (1) modes. Present methodology (-), Euler-Bernoulli analytical results (...) . . . . .

5 Dispersion curves for the beam, at different frequencies: $1 \mathrm{kHz}(1), 2 \mathrm{kHz}(2), 3 \mathrm{kHz}$ (3) and $4 \mathrm{kHz}$ (4). Present methodology (-), Euler-Bernoulli analytical results (...)

6 Reflection and transmission efficiencies of the beam's coupling joint. Present methodology: reflection (-), transmission (- $)$. Analytical results: reflection $\left(^{*}\right)$, transmission (o). Conservation of energy (...) . . . . . . . . . . . .

7 Reflection coefficient of the beam's coupling joint. Present methodology (-o), Analytical results $(*) \ldots \ldots \ldots \ldots \ldots$

8 Schematic of an orthotropic beam defined as two healthy substructures coupled through a damaged substructure. Different damage types considered are illustrated

9 Dispersion curves of the orthotropic beam: bending mode (-.), torsional mode (-) and longitudinal mode $(-) \ldots \ldots \ldots \ldots \ldots$

10 Bending wave reflection coefficient from the cracked orthotropic beam's joint. Crack severity ratios: 0.4 (-o), 0.5 (-x), 0.6 (-.), 0.7 (...), $0.8(--)$ and $0.9(-)$. . .

11 Longitudinal wave reflection coefficient from the cracked orthotropic beam's joint. Crack severity ratios: $0.1\left(\left(^{*}\right), 0.2(-\Delta), 0.3(-+), 0.4(-0), 0.5(-x), 0.6(-), 0.7\right.$. $(\ldots), 0.8(-)$ and $0.9(-) \ldots \ldots \ldots \ldots$

12 Bending wave reflection coefficient from the orthotropic laminate with notch, for various severity ratios of notch $0.1(-*), 0.2(-\Delta), 0.3(-+), 0.4(-0), 0.5(-x), 0.6$ $(-),. 0.7(\ldots), 0.8(--)$ and $0.9(-) \ldots \ldots \ldots \ldots$

13 Longitudinal wave reflection coefficient from the orthotropic laminate with notch, for various severity ratios of notch $0.1(-*), 0.2(-\Delta), 0.3(-+), 0.4(-0), 0.5(-x), 0.6$ $(-),. 0.7(\ldots), 0.8(--)$ and $0.9(-) \ldots \ldots \ldots \ldots$

14 Bending wave reflection coefficient from the orthotropic laminate with interlaminar delamination, for various severity ratios of delamination $0.1\left(-^{*}\right), 0.2(-\Delta), 0.3$ $(-+), 0.4(-0), 0.5(-x), 0.6(-),. 0.7(\ldots), 0.8(--)$ and $0.9(-) \ldots \ldots . . . .$.

15 Longitudinal wave reflection coefficient from the orthotropic laminate with interlaminar delamination, for various severity ratios of delamination $0.1(-*), 0.2(-\Delta)$, $0.3(-+), 0.4(-0), 0.5(-x), 0.6(-),. 0.7(\ldots), 0.8(--)$ and $0.9(-) \ldots \ldots$. . . . .

16 Bending wave reflection coefficient as a function of damage severity (for different damage types) within the orthotropic beam . . . . . . . . . . . . . .

17 Longitudinal wave reflection coefficient as a function of damage severity (for different damage types) within the orthotropic beam . . . . . . . . . . . . . 
18 Schematic of a sandwich laminate defined as two healthy substructures coupled through a damaged substructure. Different damage types considered are illustrated

19 Dispersion curves for the sandwich laminate: bending mode (-.), torsional mode (- -) and longitudinal mode $(-) \ldots \ldots \ldots \ldots$

20 Bending wave reflection coefficient from the sandwich laminate with crack, for various severity ratios of crack $0.1(-*), 0.2(-\Delta), 0.3(-+), 0.4(-0), 0.5(-x), 0.6$ $(-),. 0.7(\ldots), 0.8(-)$ and $0.9(-) \ldots \ldots \ldots \ldots$

21 Longitudinal wave reflection coefficient from the sandwich laminate with crack, for various severity ratios of crack $0.1(-*), 0.2(-\Delta), 0.3(-+), 0.4(-0), 0.5(-x), 0.6$ $(-),. 0.7(\ldots), 0.8(--)$ and $0.9(-) \ldots \ldots \ldots \ldots$

22 Bending wave reflection coefficient from the sandwich laminate with notch, for various severity ratios of notch $0.1(-*), 0.2(-\Delta), 0.3(-+), 0.4(-0), 0.5(-x), 0.6$ $(-),. 0.7(\ldots), 0.8(-)$ and $0.9(-) \ldots \ldots \ldots \ldots$

23 Longitudinal wave reflection coefficient from the sandwich laminate with notch, for various severity ratios of notch $0.1(-*), 0.2(-\Delta), 0.3(-+), 0.4(-0), 0.5(-x), 0.6$ $(-),. 0.7(\ldots), 0.8(-)$ and $0.9(-) \ldots \ldots \ldots \ldots$

24 Bending wave reflection coefficient from the sandwich laminate with skin-core interlaminar delamination, for various severity ratios of delamination $0.1\left({ }^{*}\right), 0.2$ $(-\Delta), 0.3(-+), 0.4(-0), 0.5(-x), 0.6(-),. 0.7(\ldots), 0.8(--)$ and $0.9(-) \ldots \ldots$. . .

25 Longitudinal wave reflection coefficient from the sandwich laminate with skincore interlaminar delamination, for various severity ratios of delamination $0.1\left(-^{*}\right)$, $0.2(-\Delta), 0.3(-+), 0.4(-0), 0.5(-x), 0.6(-),. 0.7(\ldots), 0.8(--)$ and $0.9(-)$. . . . .

26 Bending wave reflection coefficient from the sandwich laminate with core interlaminar delamination, for various severity ratios of delamination $0.1(-*), 0.2(-\Delta)$, $0.3(-+), 0.4(-0), 0.5(-x), 0.6(-),. 0.7(\ldots), 0.8(--)$ and $0.9(-) \ldots \ldots$. . . . .

27 Longitudinal wave reflection coefficient from the sandwich laminate with core interlaminar delamination, for various severity ratios of delamination $0.1(-*), 0.2$ $(-\Delta), 0.3(-+), 0.4(-0), 0.5(-x), 0.6(-),. 0.7(\ldots), 0.8(--)$ and $0.9(-) \ldots \ldots$

28 Bending wave reflection coefficient as a function of damage severity (for different damage types) within the sandwich laminate . . . . . . . . . . . .

29 Longitudinal wave reflection coefficient as a function of damage severity (for different damage types) within the sandwich laminate . . . . . . . . . . .

30 Finite element modelling of damage using the node duplication method: (a) damaged structural segment, (b) node duplication model . . . . . . . . . . . 


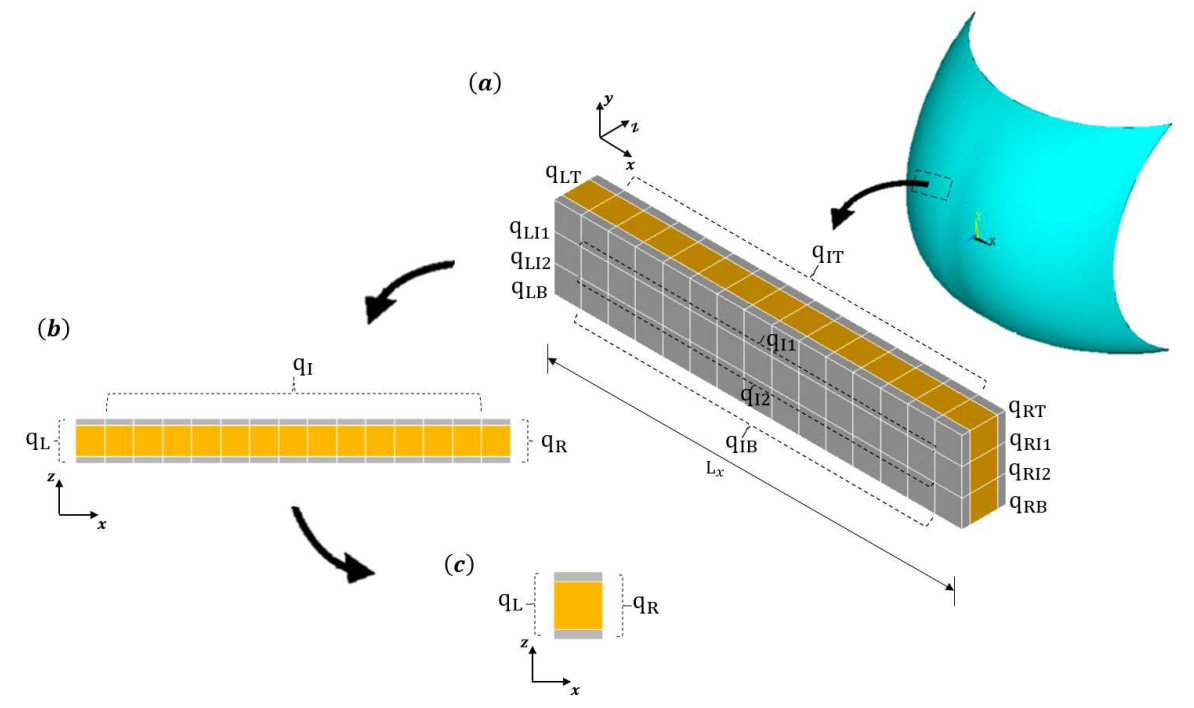

Figure 1: Periodic FE-modelled segment of an arbitrarily layered composite structure 


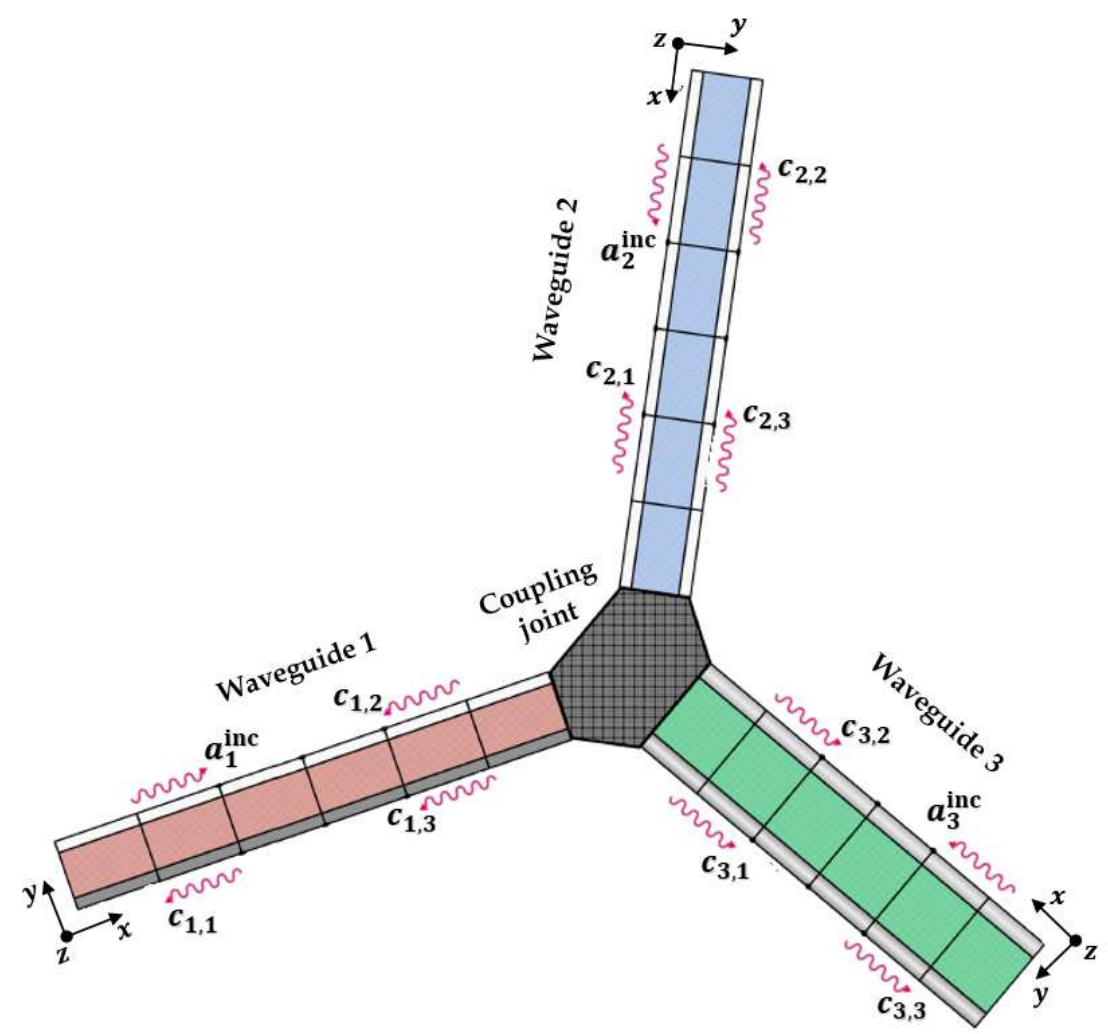

Figure 2: Periodic waveguides connected through a coupling joint which carries localised damage. A positive going wave of amplitude $\mathbf{a}_{n}^{\text {inc }}$ impinging on the joint from $n$th waveguide will give rise to wave of reflection coefficient $\mathbf{c}_{n, n}$ in the $n$th waveguide and wave of transmission coefficient $\mathbf{c}_{k, n}$ in the $k$ th waveguide 


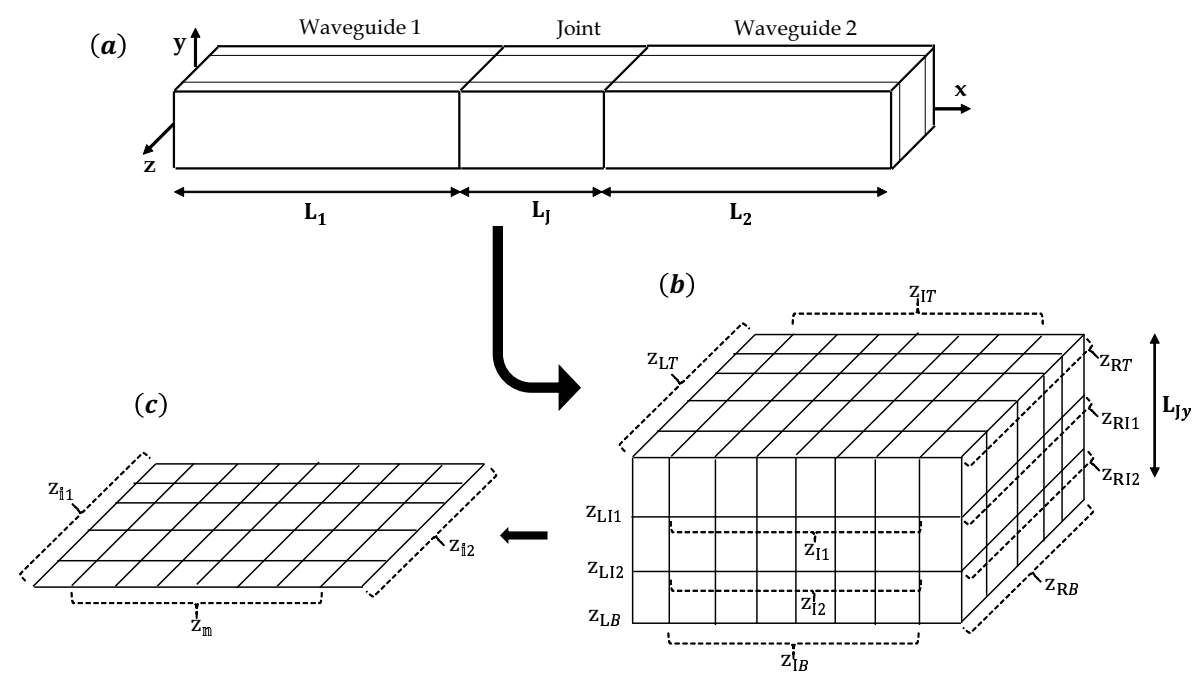

Figure 3: PST formulation of a typical fully FE-modelled coupling joint 


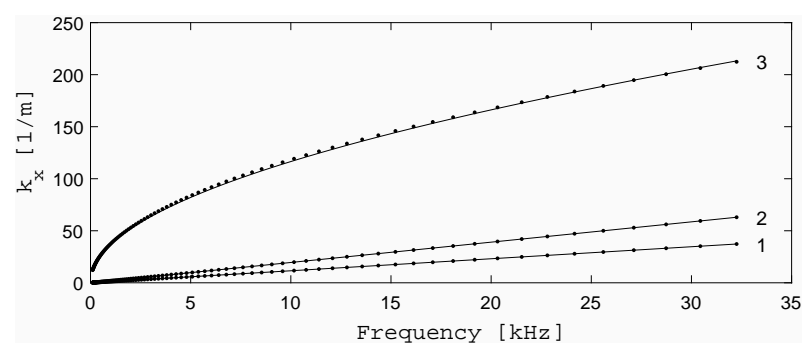

(a) at $k_{y}=0 m^{-1}$

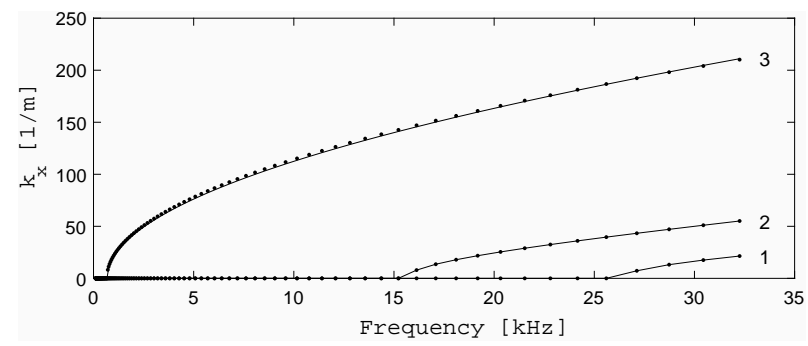

(c) at $k_{y}=30 m^{-1}$

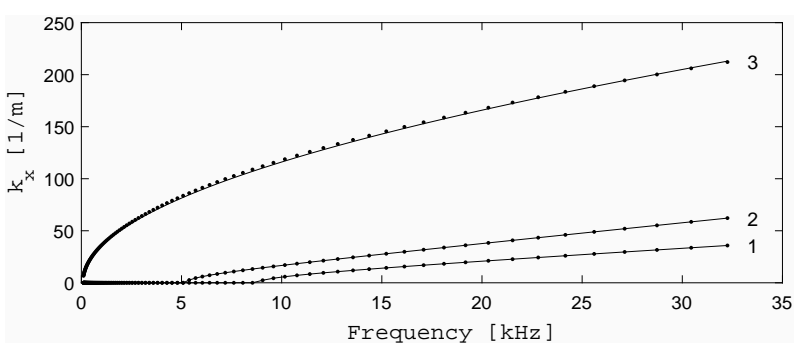

(b) at $k_{y}=10 m^{-1}$

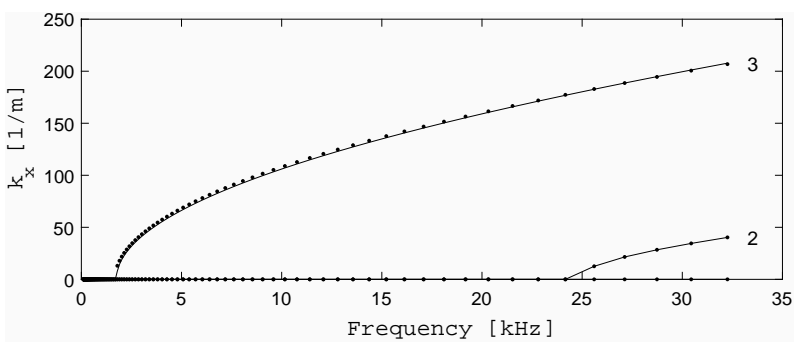

(d) at $k_{y}=50 m^{-1}$

Figure 4: Dispersion curves for the beam: bending (3), torsional (2) and longitudinal (1) modes. Present methodology $(-)$, Euler-Bernoulli analytical results (...) 


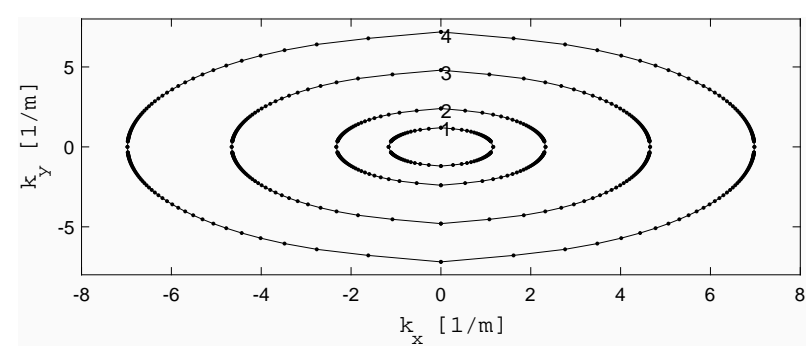

(a) longitudinal wave

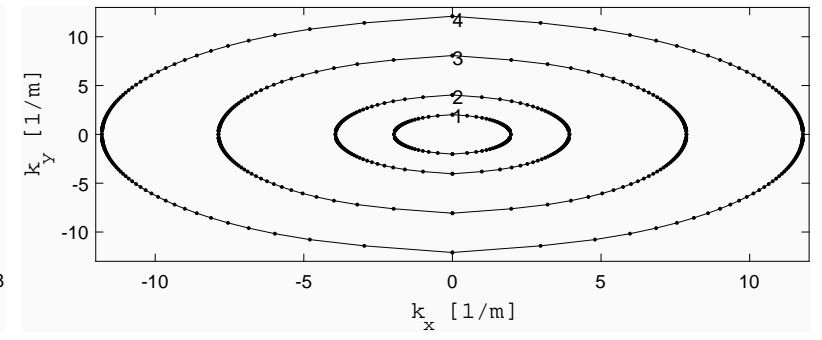

(b) torsional wave

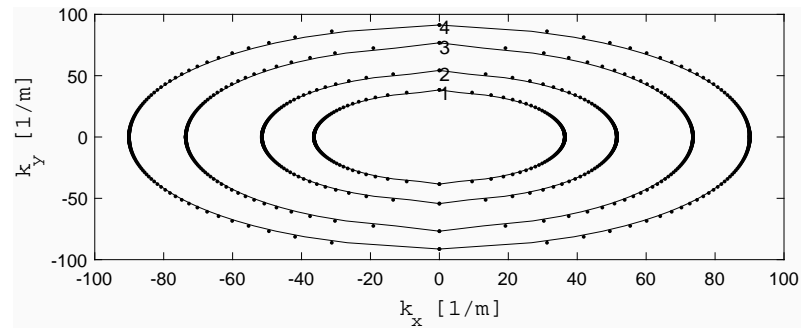

(c) bending wave

Figure 5: Dispersion curves for the beam, at different frequencies: $1 \mathrm{kHz}(1), 2 \mathrm{kHz}(2), 3 \mathrm{kHz}$ (3) and $4 \mathrm{kHz}$ (4). Present methodology (-), Euler-Bernoulli analytical results (...) 


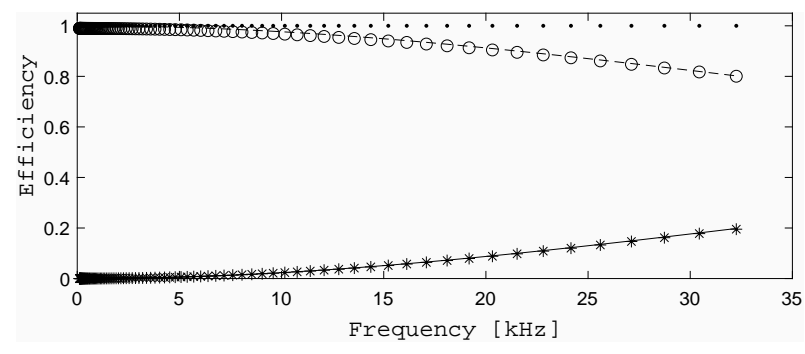

(a) longitudinal wave

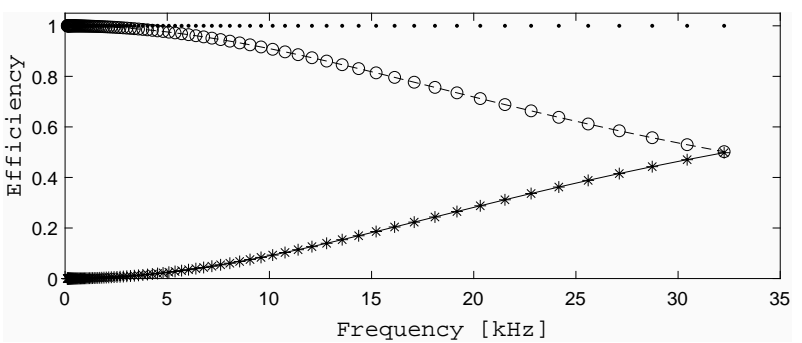

(b) torsional wave

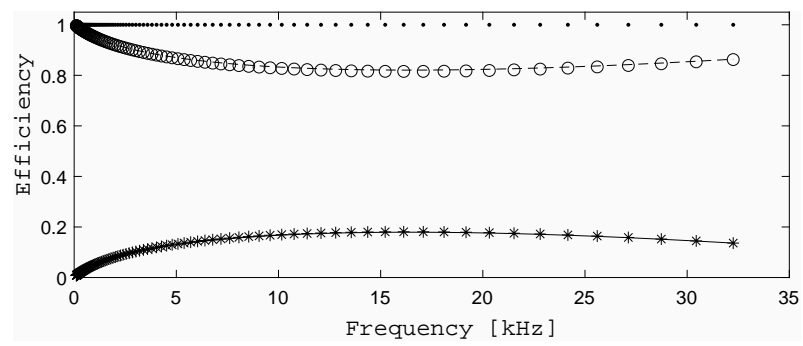

(c) bending wave

Figure 6: Reflection and transmission efficiencies of the beam's coupling joint. Present methodology: reflection (-), transmission (- -). Analytical results: reflection (*), transmission (o). Conservation of energy (...) 


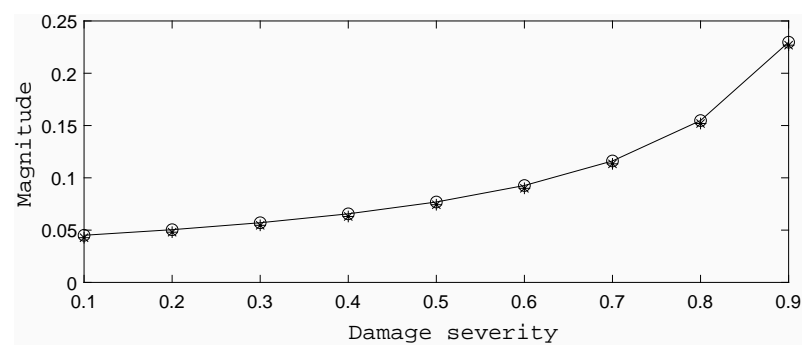

(a) longitudinal wave

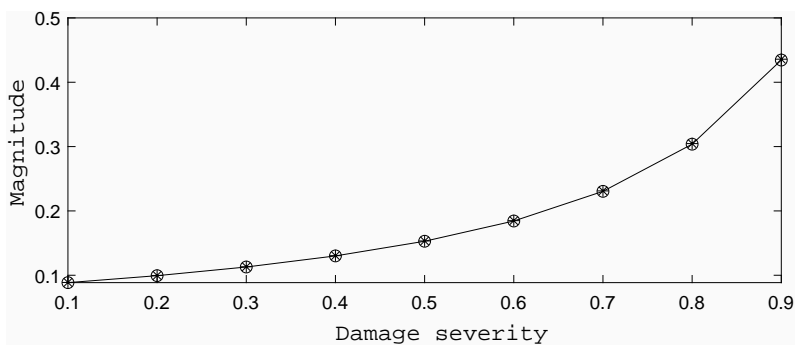

(b) torsional wave

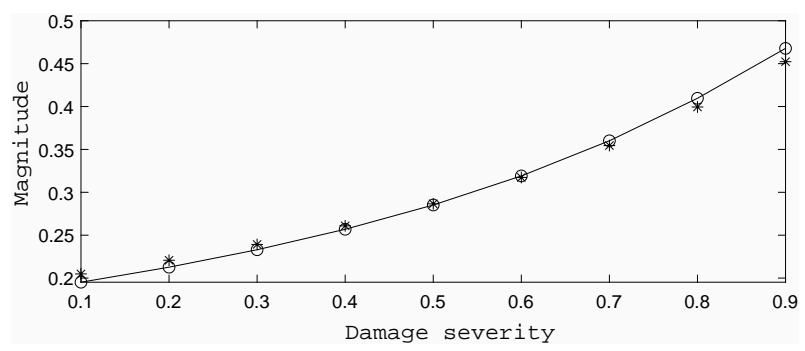

(c) bending wave

Figure 7: Reflection coefficient of the beam's coupling joint. Present methodology (-o), Analytical results (*) 


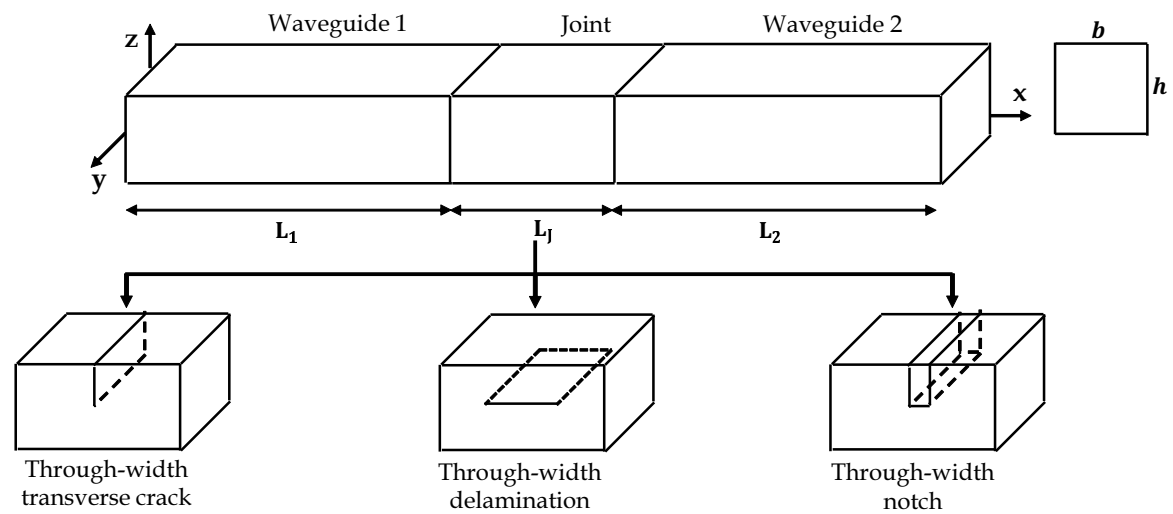

Figure 8: Schematic of an orthotropic beam defined as two healthy substructures coupled through a damaged substructure. Different damage types considered are illustrated 


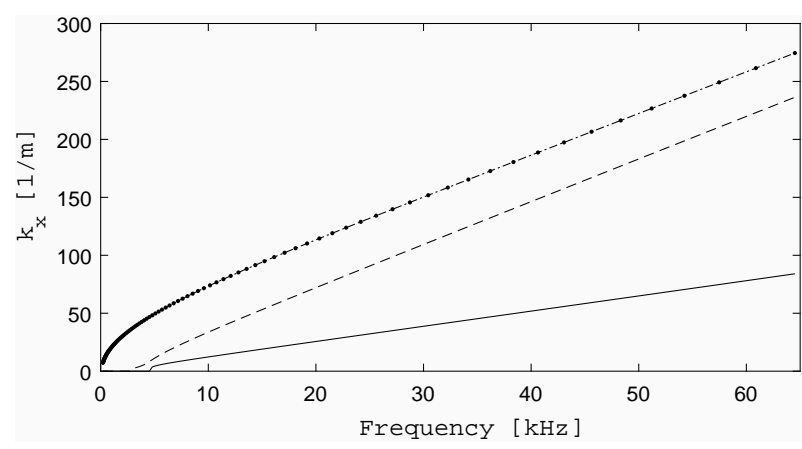

(a) $\left(\omega, k_{x}\right)$ plane for $k_{y}=12 \mathrm{~m}^{-1}$

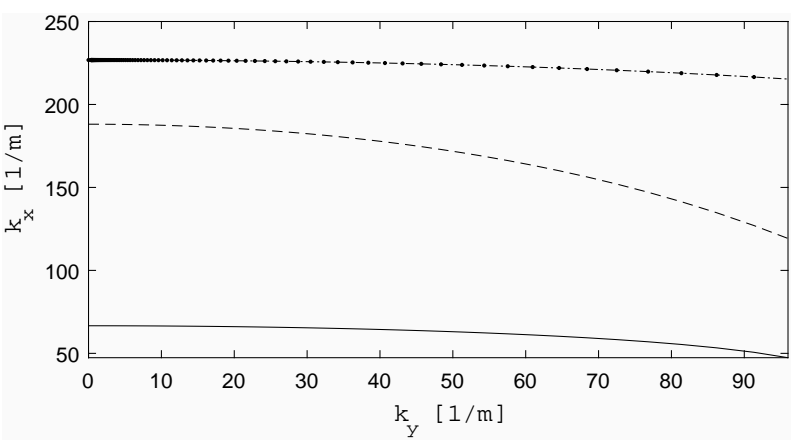

(b) $\left(k_{y}, k_{x}\right)$ plane for $\omega=50 \mathrm{kHz}$

Figure 9: Dispersion curves of the orthotropic beam: bending mode (-.), torsional mode (- -) and longitudinal mode $(-)$ 


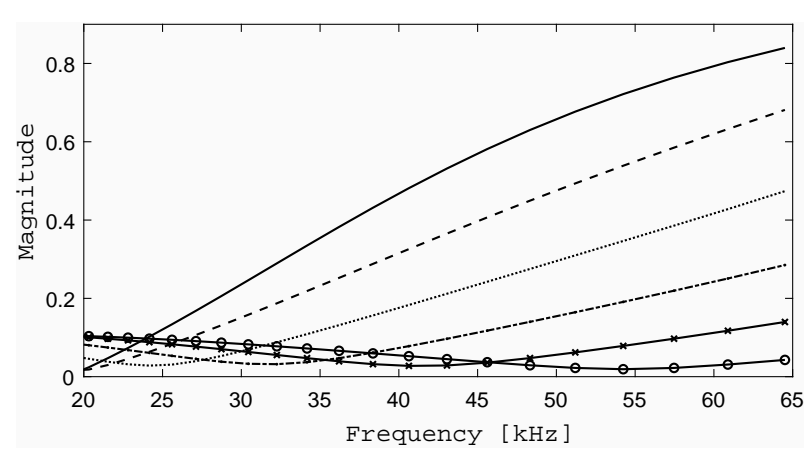

(a) w.r.t $\omega$

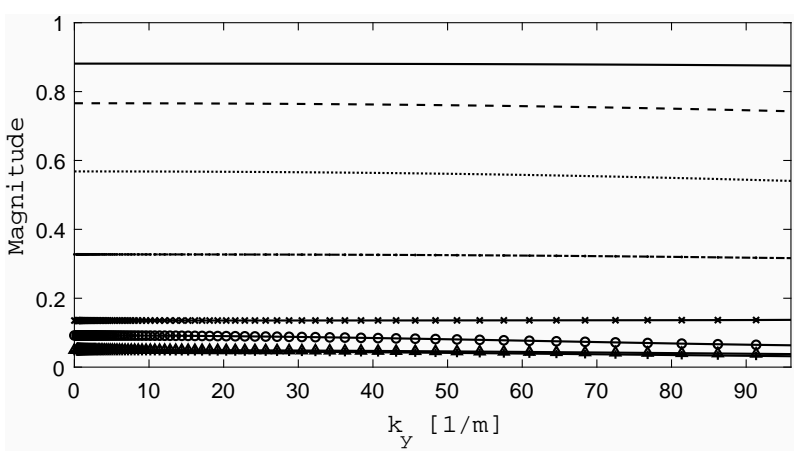

(b) w.r.t. $k_{y}$

Figure 10: Bending wave reflection coefficient from the cracked orthotropic beam's joint. Crack severity ratios: 0.4 $(-0), 0.5(-x), 0.6(-),. 0.7(\ldots), 0.8(-)$ and $0.9(-)$ 


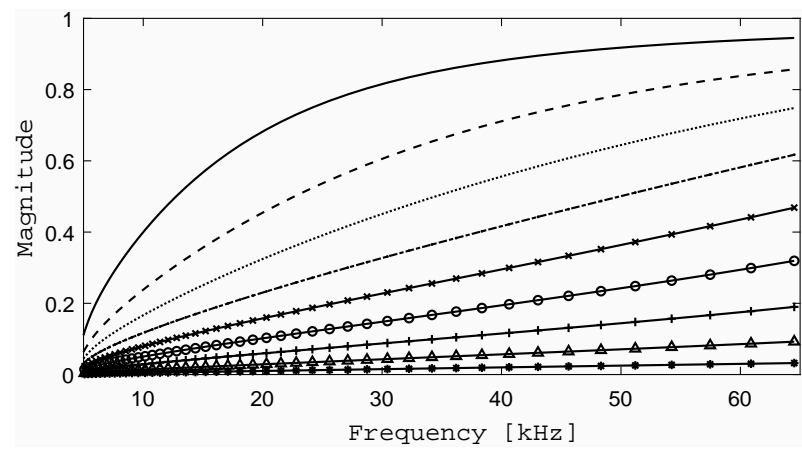

(a) w.r.t $\omega$

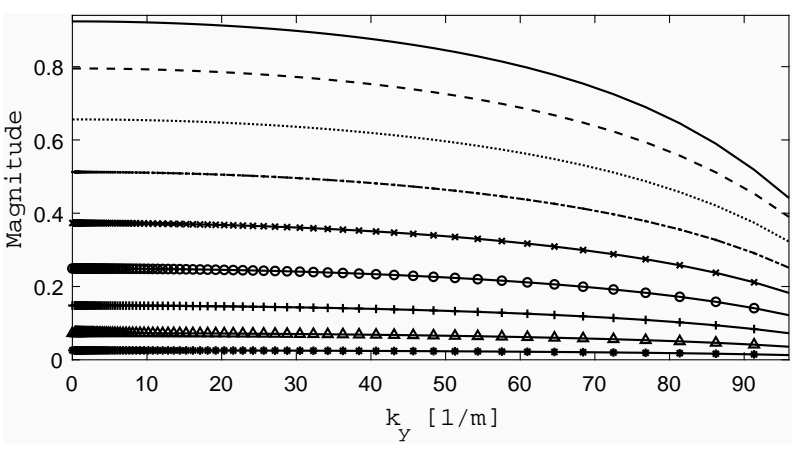

(b) w.r.t. $k_{y}$

Figure 11: Longitudinal wave reflection coefficient from the cracked orthotropic beam's joint. Crack severity ratios: $0.1(-*), 0.2(-\Delta), 0.3(-+), 0.4(-0), 0.5(-x), 0.6(-),. 0.7(\ldots), 0.8(--)$ and $0.9(-)$ 


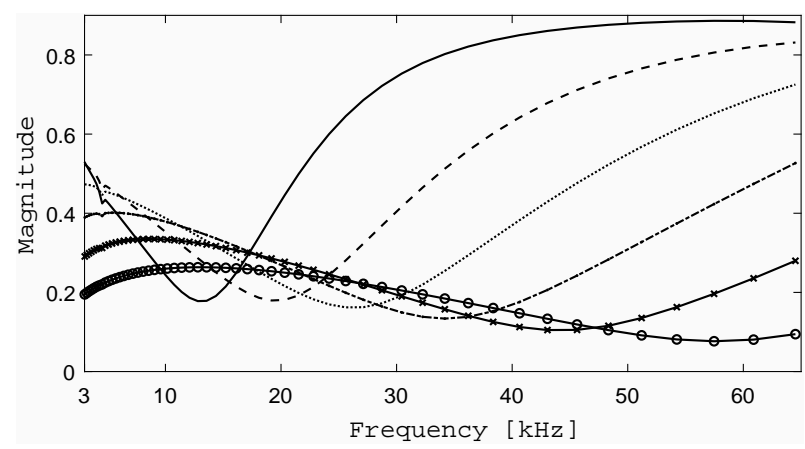

(a) w.r.t $\omega$

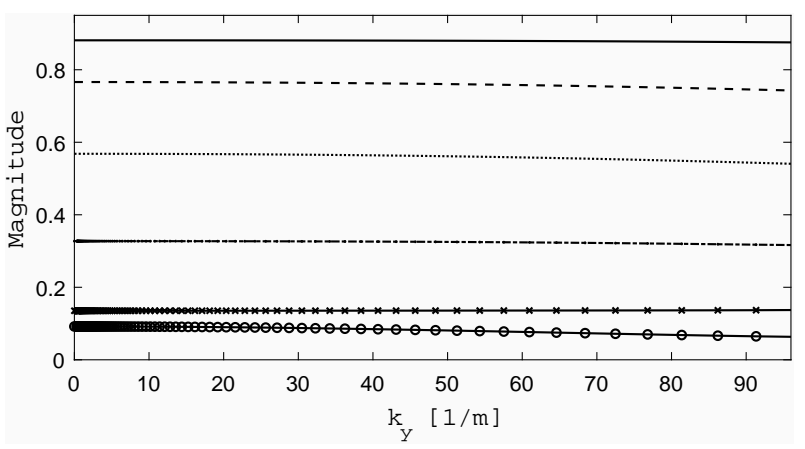

(b) w.r.t. $k_{y}$

Figure 12: Bending wave reflection coefficient from the orthotropic laminate with notch, for various severity ratios of notch $0.1(-*), 0.2(-\Delta), 0.3(-+), 0.4(-0), 0.5(-x), 0.6(-),. 0.7(\ldots), 0.8(--)$ and $0.9(-)$ 


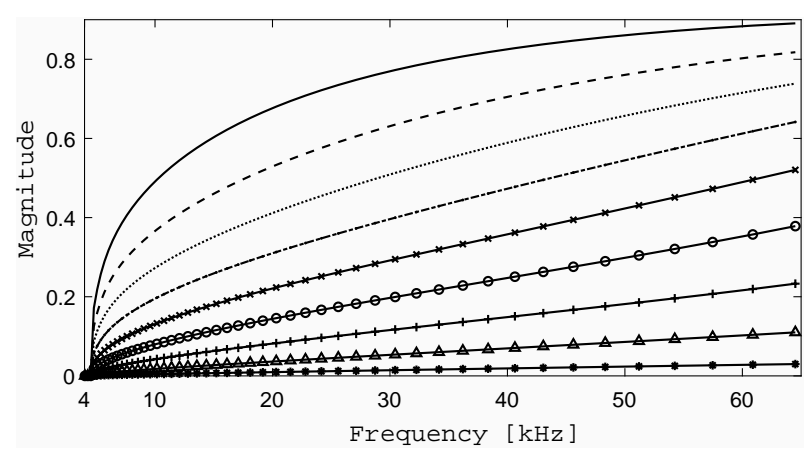

(a) w.r.t $\omega$

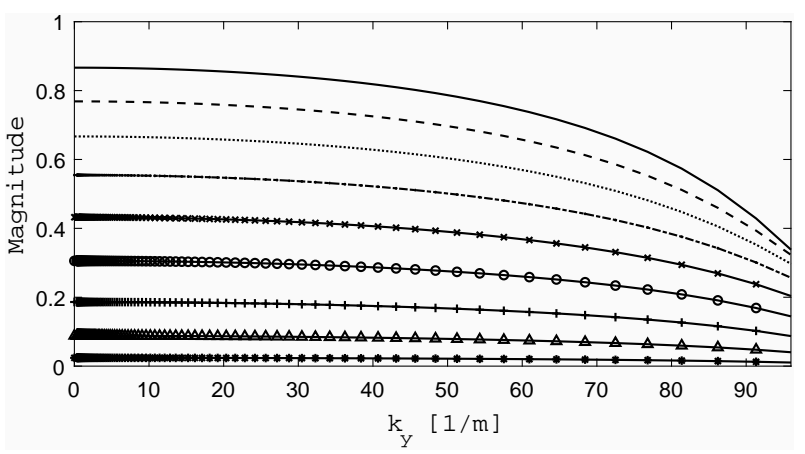

(b) w.r.t. $k_{y}$

Figure 13: Longitudinal wave reflection coefficient from the orthotropic laminate with notch, for various severity ratios of notch $0.1(-*), 0.2(-\Delta), 0.3(-+), 0.4(-0), 0.5(-x), 0.6(-),. 0.7(\ldots), 0.8(--)$ and $0.9(-)$ 


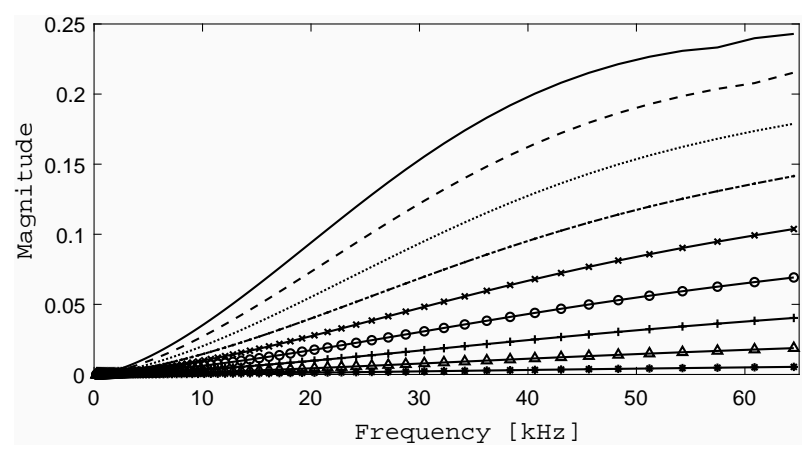

(a) w.r.t $\omega$

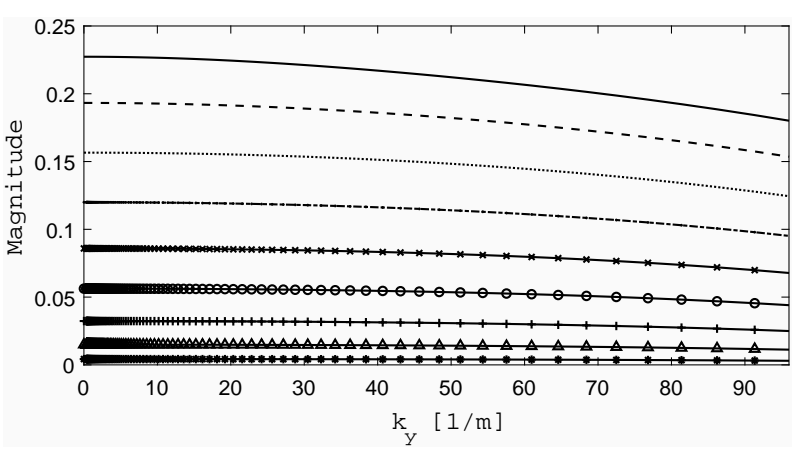

(b) w.r.t. $k_{y}$

Figure 14: Bending wave reflection coefficient from the orthotropic laminate with interlaminar delamination, for various severity ratios of delamination $0.1\left(-^{*}\right), 0.2(-\Delta), 0.3(-+), 0.4(-0), 0.5(-x), 0.6(-),. 0.7(\ldots), 0.8(--)$ and 0.9 $(-)$ 


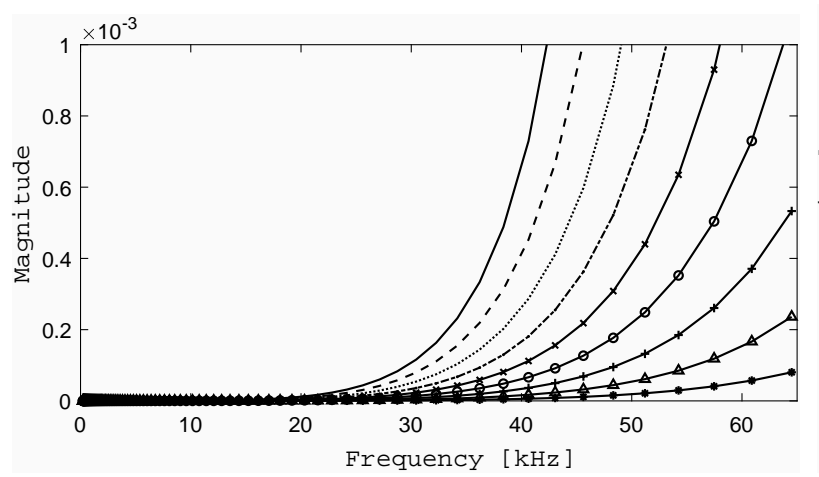

(a) w.r.t $\omega$

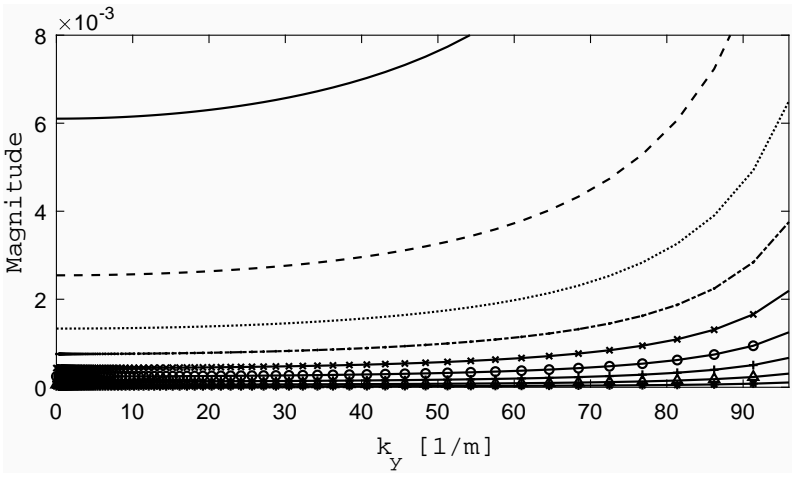

(b) w.r.t. $k_{y}$

Figure 15: Longitudinal wave reflection coefficient from the orthotropic laminate with interlaminar delamination, for various severity ratios of delamination $0.1(-*), 0.2(-\Delta), 0.3(-+), 0.4(-0), 0.5(-x), 0.6(-),. 0.7(\ldots), 0.8(--)$ and 0.9 $(-)$ 


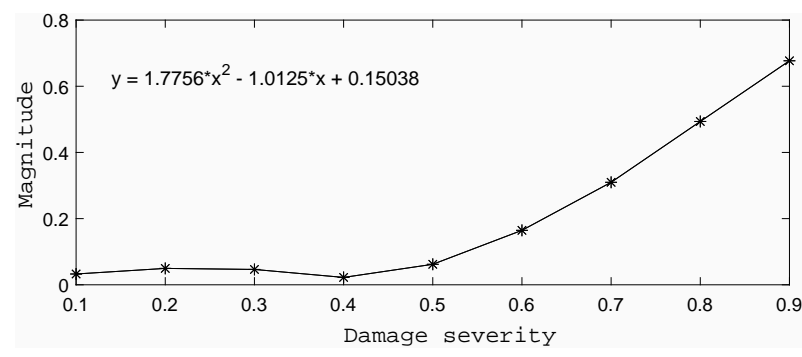

(a) crack

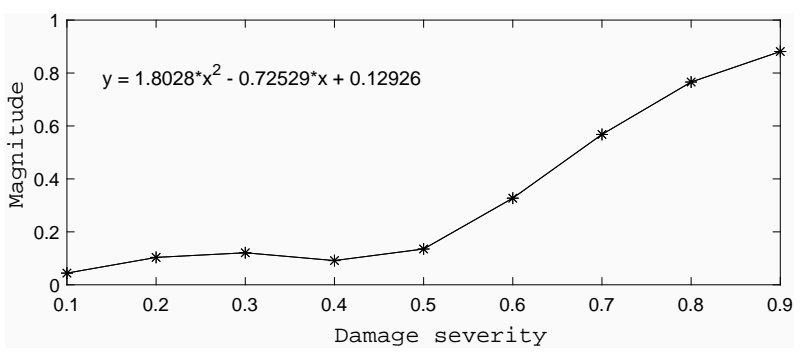

(b) notch

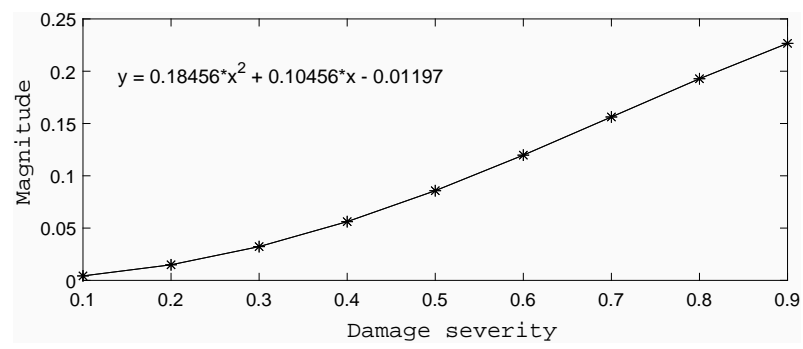

(c) interlaminar delamination

Figure 16: Bending wave reflection coefficient as a function of damage severity (for different damage types) within the orthotropic beam 


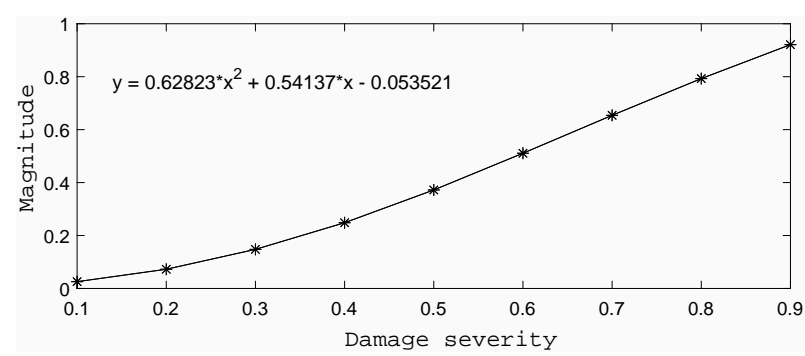

(a) crack

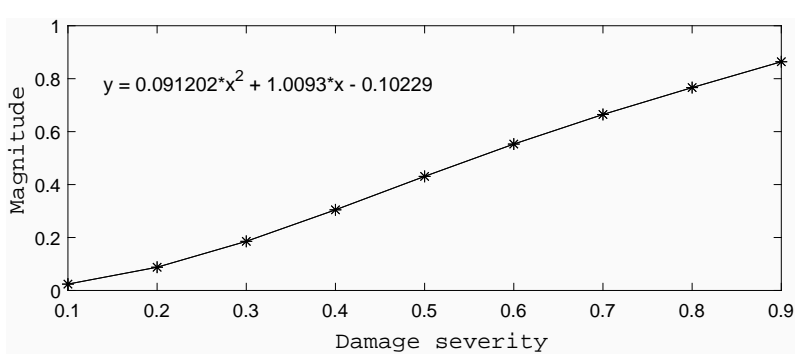

(b) notch

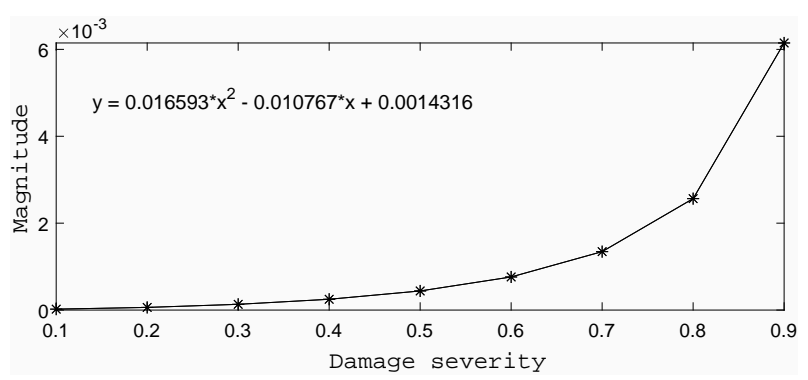

(c) interlaminar delamination

Figure 17: Longitudinal wave reflection coefficient as a function of damage severity (for different damage types) within the orthotropic beam 


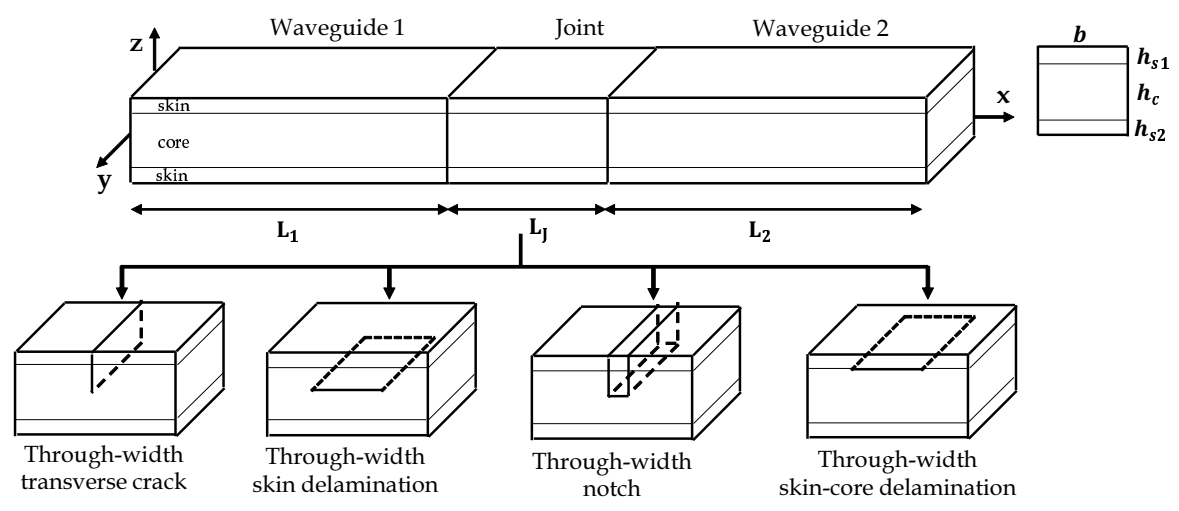

Figure 18: Schematic of a sandwich laminate defined as two healthy substructures coupled through a damaged substructure. Different damage types considered are illustrated 


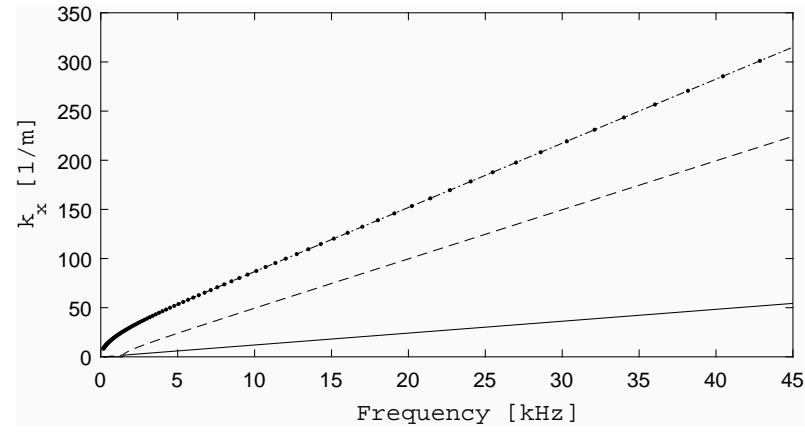

(a) $\left(\omega, k_{x}\right)$ plane

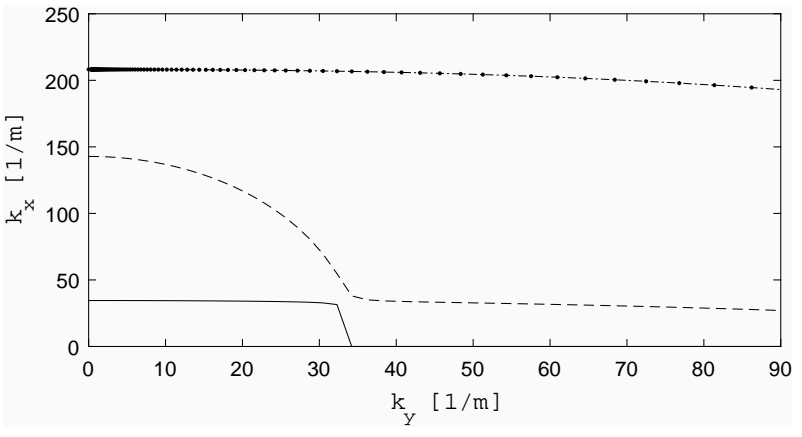

(b) $\left(k_{y}, k_{x}\right)$ plane

Figure 19: Dispersion curves for the sandwich laminate: bending mode (-.), torsional mode (- -) and longitudinal mode (-) 


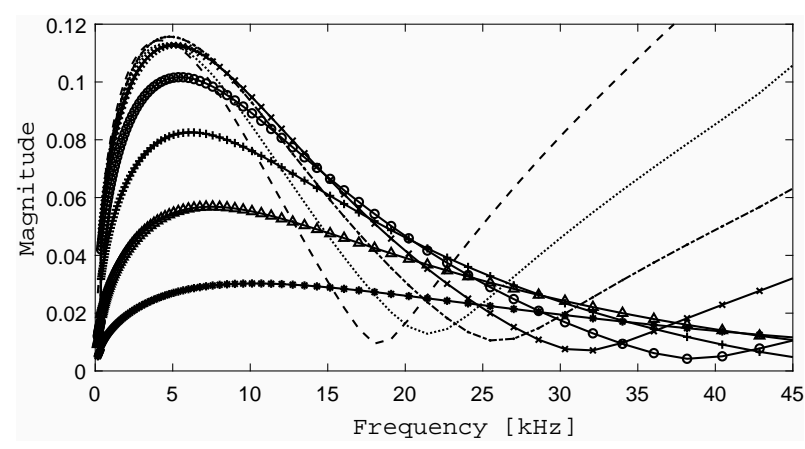

(a) w.r.t $\omega$

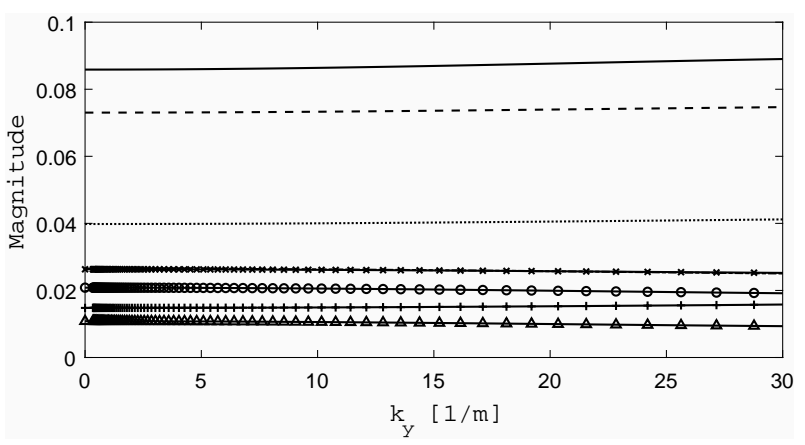

(b) w.r.t. $k_{y}$

Figure 20: Bending wave reflection coefficient from the sandwich laminate with crack, for various severity ratios of crack $0.1(-*), 0.2(-\Delta), 0.3(-+), 0.4(-0), 0.5(-x), 0.6(-),. 0.7(\ldots), 0.8(--)$ and $0.9(-)$ 


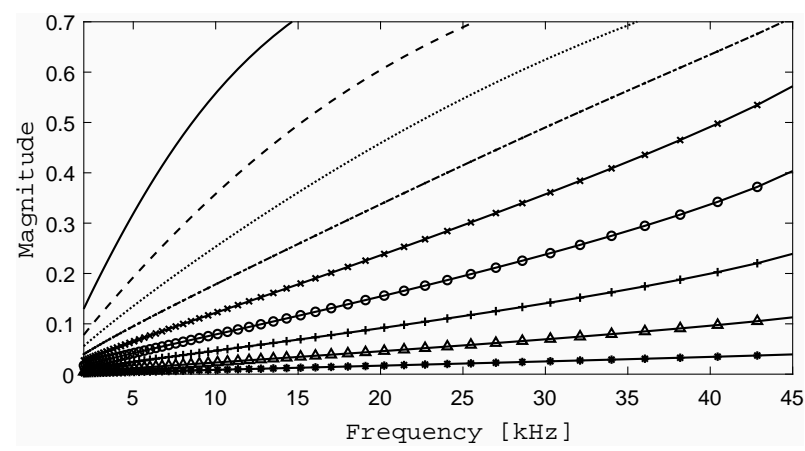

(a) w.r.t $\omega$

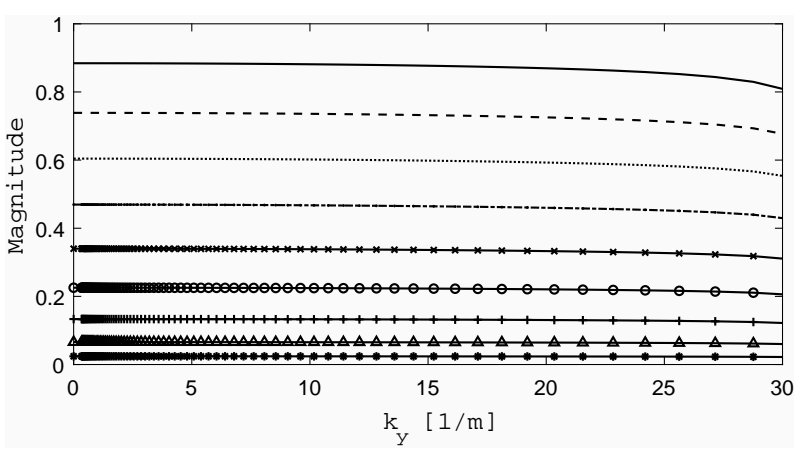

(b) w.r.t. $k_{y}$

Figure 21: Longitudinal wave reflection coefficient from the sandwich laminate with crack, for various severity ratios of crack $0.1(-*), 0.2(-\Delta), 0.3(-+), 0.4(-0), 0.5(-x), 0.6(-),. 0.7(\ldots), 0.8(--)$ and $0.9(-)$ 


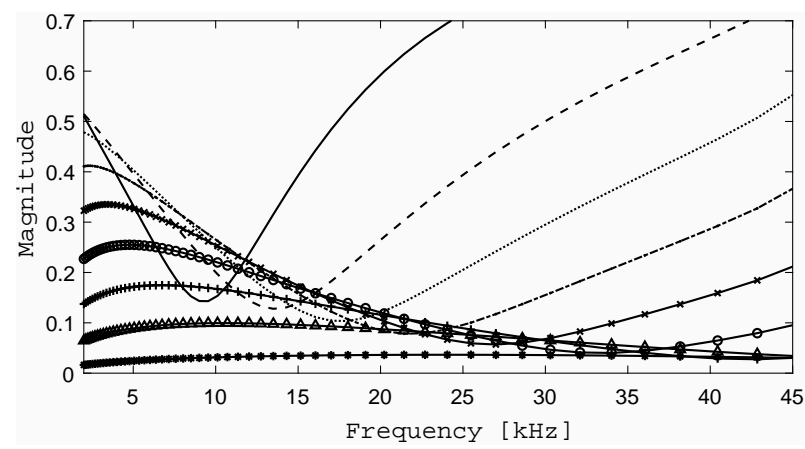

(a) w.r.t $\omega$

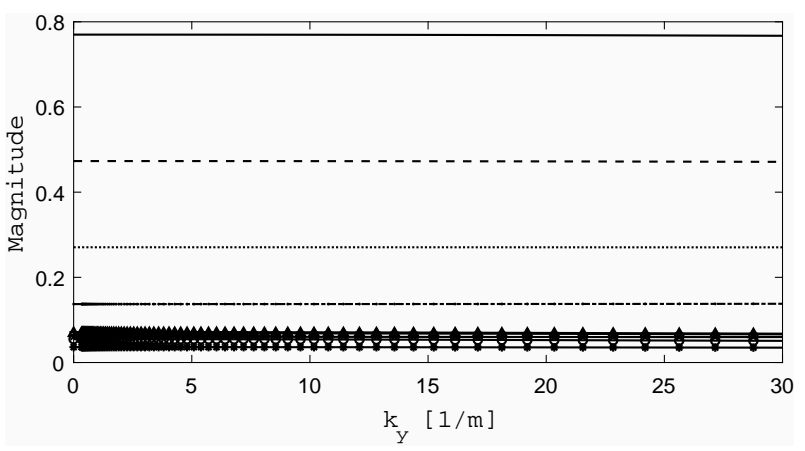

(b) w.r.t. $k_{y}$

Figure 22: Bending wave reflection coefficient from the sandwich laminate with notch, for various severity ratios of notch $0.1\left(\right.$ (*) $^{*} 0.2(-\Delta), 0.3(-+), 0.4(-0), 0.5(-x), 0.6(-),. 0.7(\ldots), 0.8(--)$ and $0.9(-)$ 


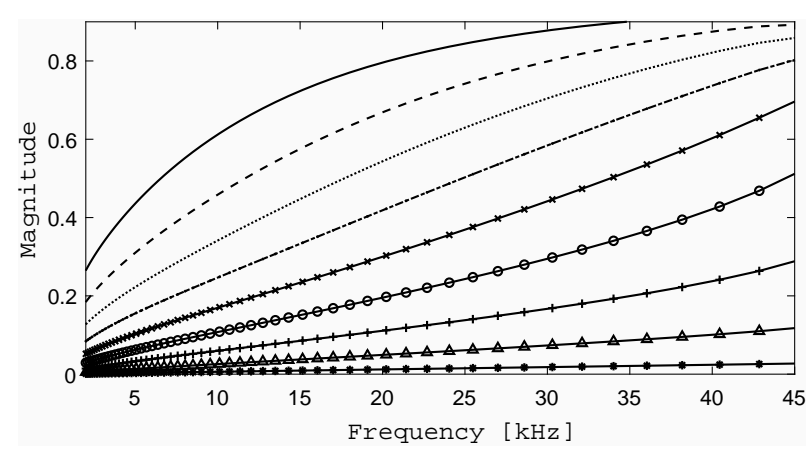

(a) w.r.t $\omega$

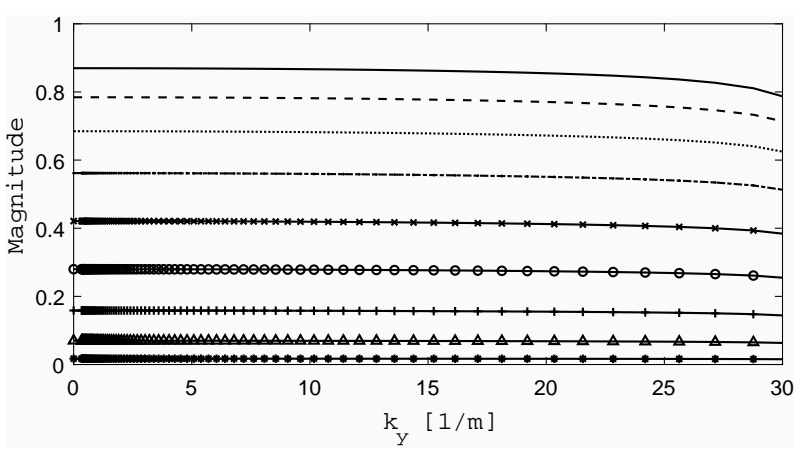

(b) w.r.t. $k_{y}$

Figure 23: Longitudinal wave reflection coefficient from the sandwich laminate with notch, for various severity ratios of notch $0.1(-*), 0.2(-\Delta), 0.3(-+), 0.4(-0), 0.5(-x), 0.6(-),. 0.7(\ldots), 0.8(--)$ and $0.9(-)$ 


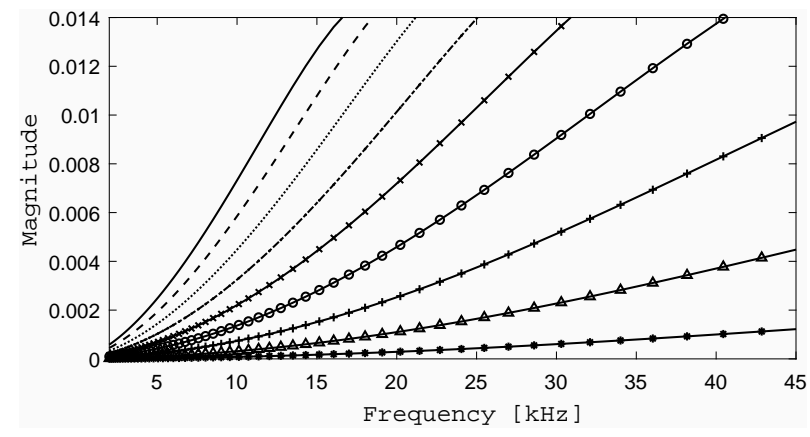

(a) w.r.t $\omega$

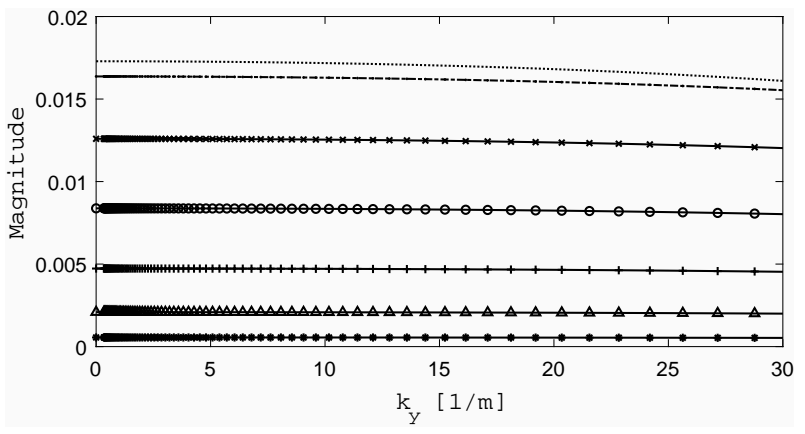

(b) w.r.t. $k_{y}$

Figure 24: Bending wave reflection coefficient from the sandwich laminate with skin-core interlaminar delamination, for various severity ratios of delamination $0.1\left(\left(^{*}\right), 0.2(-\Delta), 0.3(-+), 0.4(-0), 0.5(-x), 0.6(-),. 0.7(\ldots), 0.8(--)\right.$ and $0.9(-)$ 


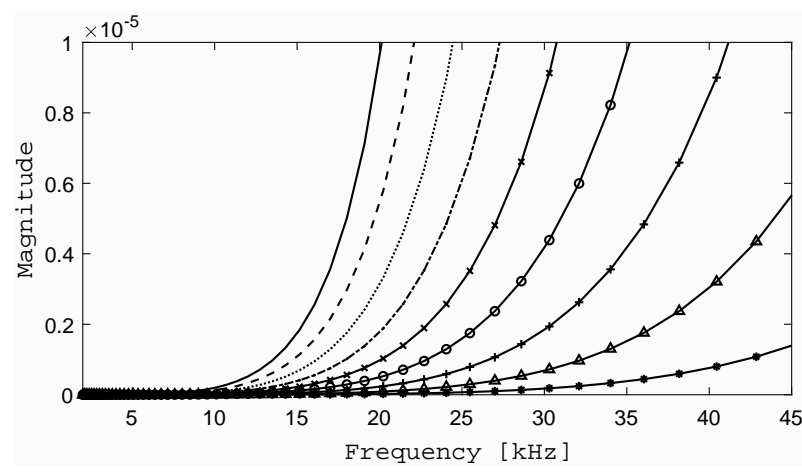

(a) w.r.t $\omega$

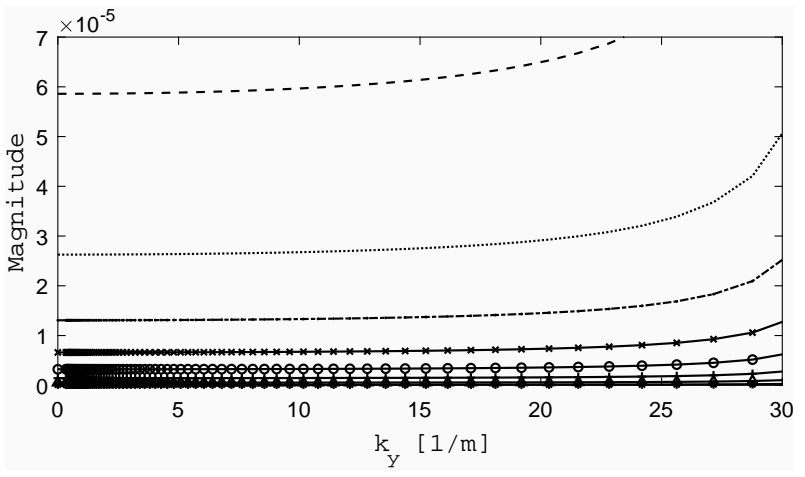

(b) w.r.t. $k_{y}$

Figure 25: Longitudinal wave reflection coefficient from the sandwich laminate with skin-core interlaminar delamination, for various severity ratios of delamination $0.1(-*), 0.2(-\Delta), 0.3(-+), 0.4(-0), 0.5(-\mathrm{x}), 0.6(-),. 0.7(\ldots), 0.8(-$ -) and $0.9(-)$ 


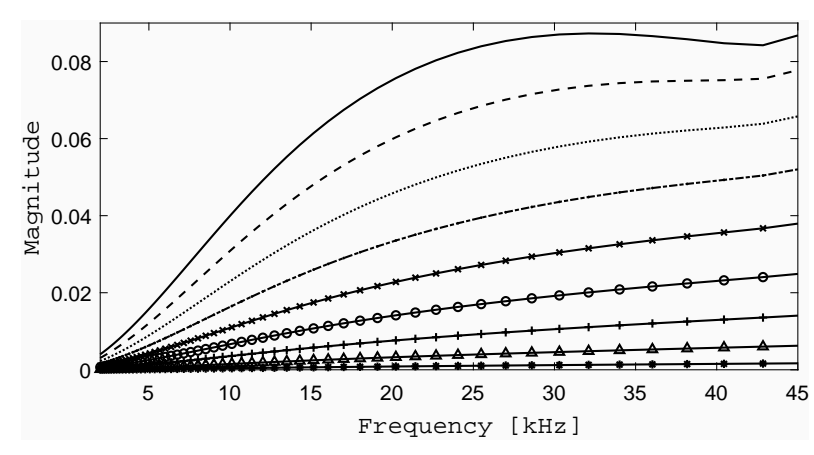

(a) w.r.t $\omega$

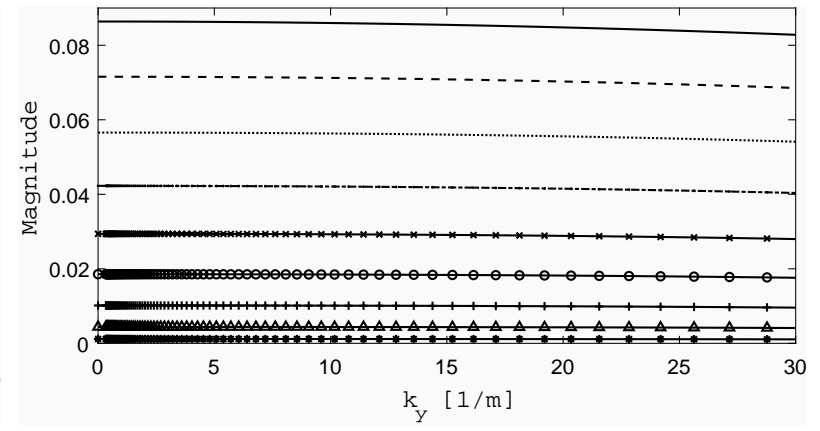

(b) w.r.t. $k_{y}$

Figure 26: Bending wave reflection coefficient from the sandwich laminate with core interlaminar delamination, for various severity ratios of delamination $0.1\left(\left(^{*}\right), 0.2(-\Delta), 0.3(-+), 0.4(-0), 0.5(-x), 0.6(-),. 0.7(\ldots), 0.8(--)\right.$ and 0.9 $(-)$ 


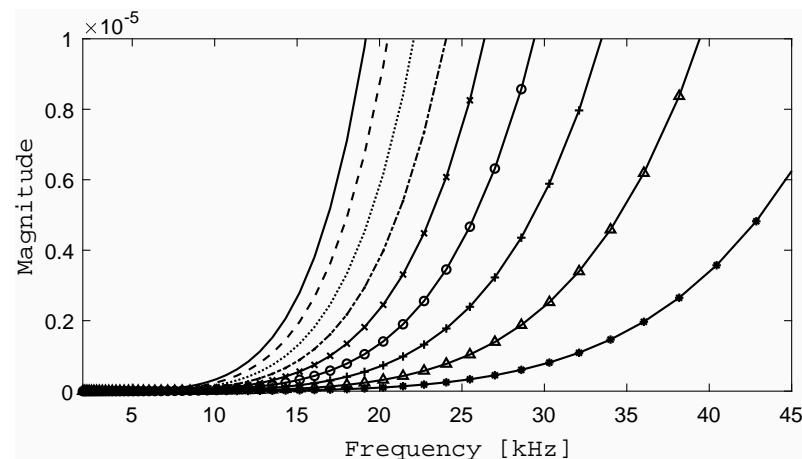

(a) w.r.t $\omega$

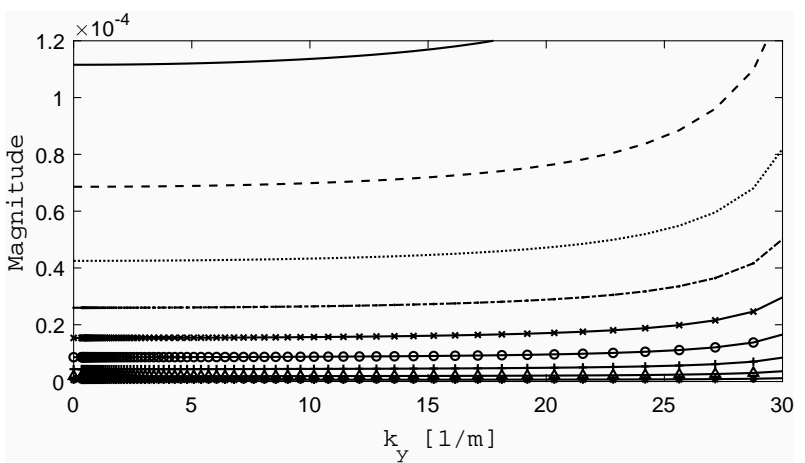

(b) w.r.t. $k_{y}$

Figure 27: Longitudinal wave reflection coefficient from the sandwich laminate with core interlaminar delamination, for various severity ratios of delamination $0.1(-*), 0.2(-\Delta), 0.3(-+), 0.4(-0), 0.5(-x), 0.6(-),. 0.7(\ldots), 0.8(--)$ and $0.9(-)$ 


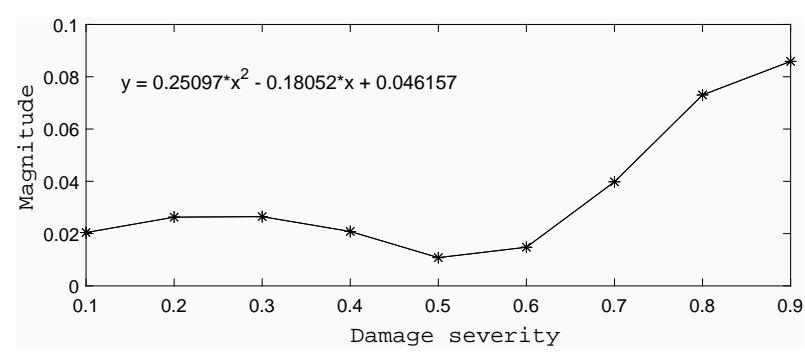

(a) crack

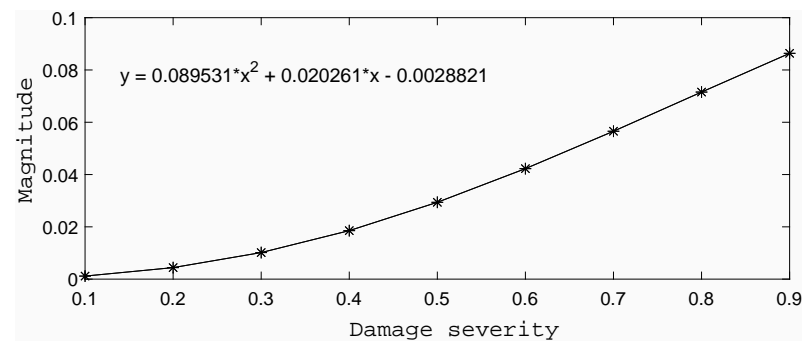

(c) core interlaminar delamination

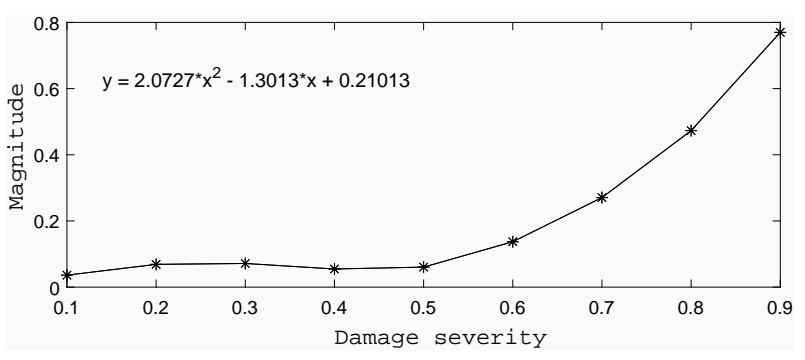

(b) notch

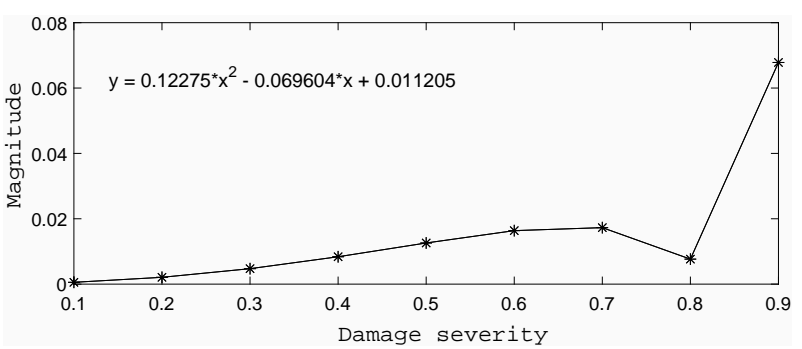

(d) skin-core interlaminar delamination

Figure 28: Bending wave reflection coefficient as a function of damage severity (for different damage types) within the sandwich laminate 


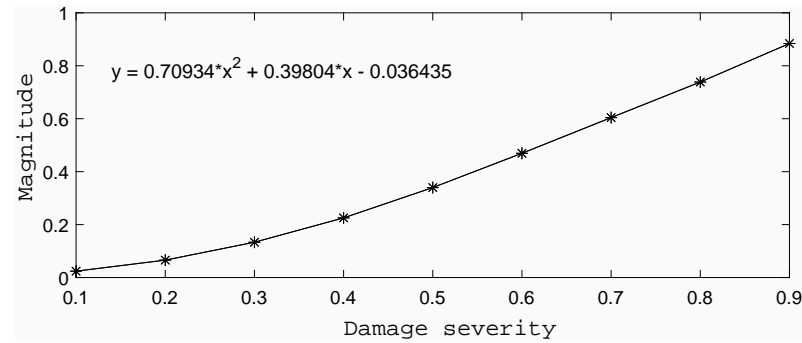

(a) crack

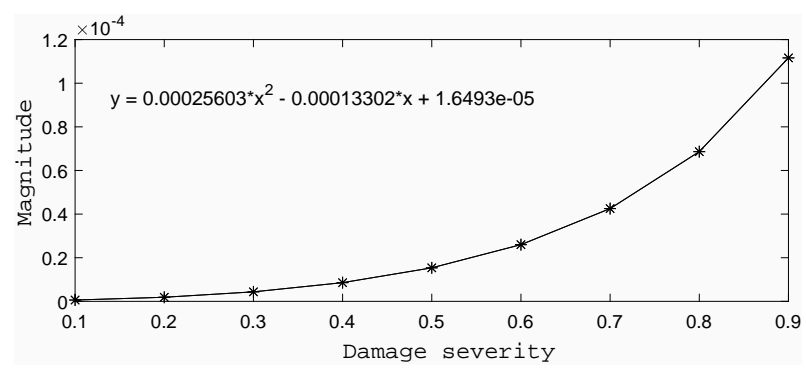

(c) core interlaminar delamination

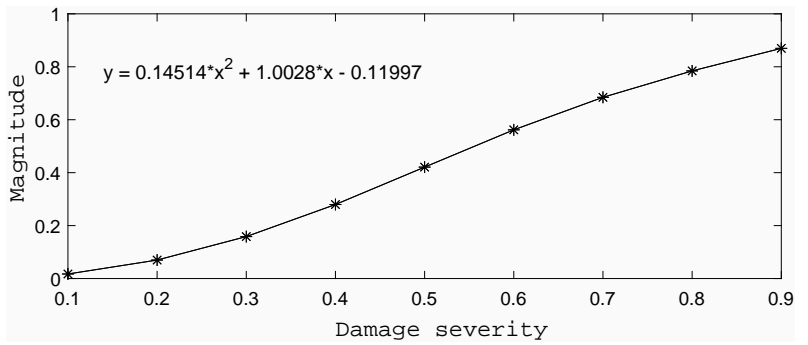

(b) notch

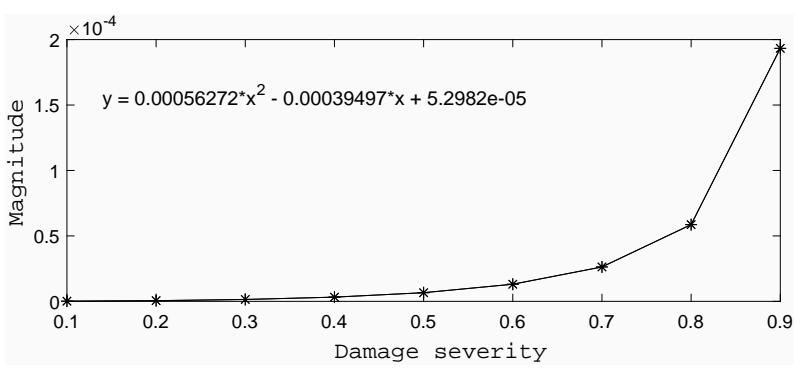

(d) skin-core interlaminar delamination

Figure 29: Longitudinal wave reflection coefficient as a function of damage severity (for different damage types) within the sandwich laminate 

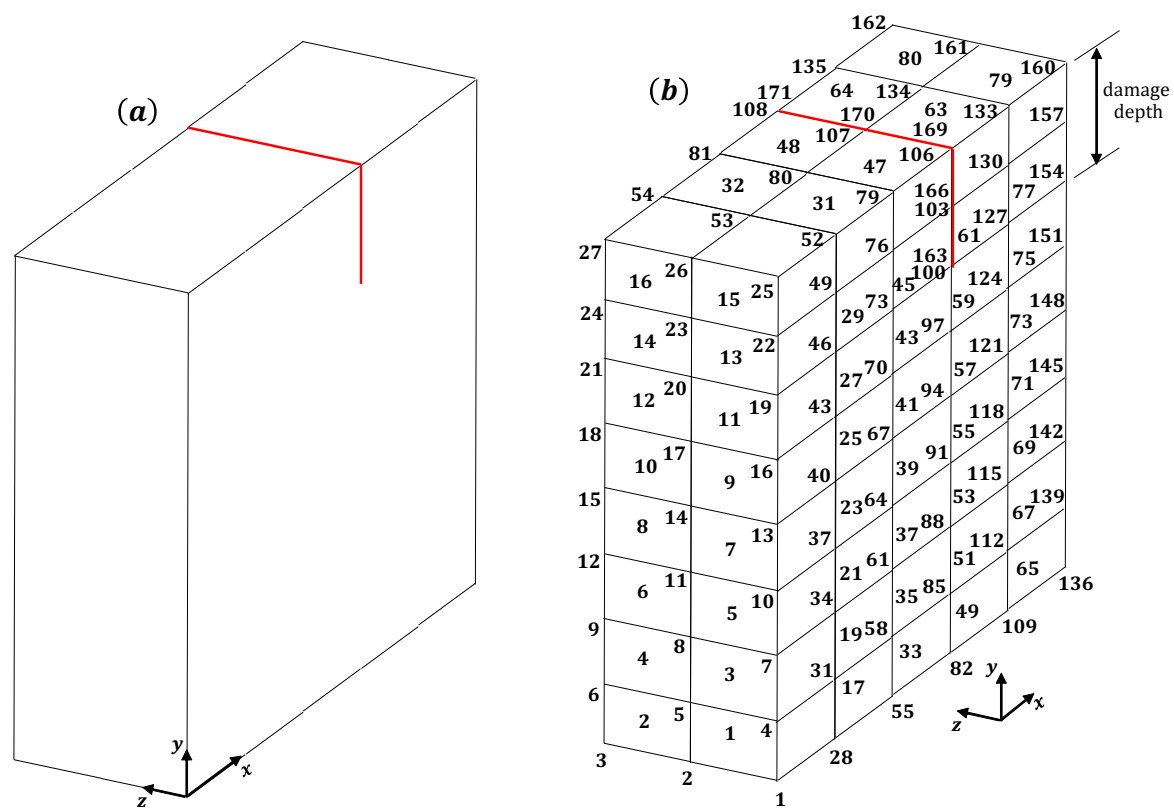

Figure 30: Finite element modelling of damage using the node duplication method: (a) damaged structural segment, (b) node duplication model 